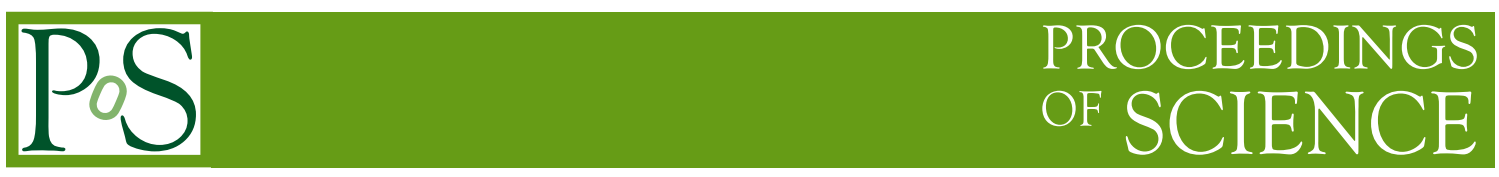

\title{
The 6-vertex model with fixed boundary conditions.
}

\author{
Nicolai Reshetikhin ${ }^{* \dagger}$ \\ Department of mathematics, University of California, Berkeley, CA 94720, USA \\ E-mail: reshetik@math.berkeley.edu

\section{Konstantin Palamarchuk} \\ Department of mathematics, University of California, Berkeley, CA 94720, USA \\ E-mail: reshetik@math.berkeley.edu
}

\begin{abstract}
We study the 6-vertex model with fixed boundary conditions. In the thermodynamical limit there is a formation of the limit shape. We collect most of the known results about the analytical properties of the free energy of the model as the function of electric fields and study the asymptotical behavior near singularities. We also study the asymptotic of limit shapes and the structure of correlation functions in the bulk
\end{abstract}

Bethe Ansatz: 75 years later

October 19-21 2006,

Brussels, Belgium

* Speaker.

${ }^{\dagger}$ A footnote may follow. 


\section{Introduction}

$*$

Ising and dimer models were among the first models in two-dimensional statistical mechanics for where the partition function and for some of the correlation functions were computed explicitly in terms of Pfaffians of certain matrices. For this reason both of these models can be regarded as theories of Gaussian discrete two dimensional fermi field. The Ising model was solved by Onsager and for dimer models the Pfaffian solution was found by Kasteleyn.

The 6-vertex model, a particular case of which is the ice model is interesting for a number of reasons. Physically, it is a model of ferro- and antiferro- electricity. It has many equivalent reformulations, one of them (which we will use) describe the 6-vertex configurations on a planar connected simply connected region in terms of stepped surfaces. One of the combinatorial reformulations of the 6-vertex model for specific value of parameters [Kup] is related to alternating sign matrices [Bres].

The 6-vertex model generalizes dimer models and can be regraded as the theory of Gaussian discrete fermions with four fermionic interaction. The partition function of the 6-vertex model with periodic boundary conditions was computed in [Lieb] using the Bethe Ansatz method.

The computation of correlation functions in the 6-vertex model is highly non-trivial and to the large degree is still a challenge. There are two known approaches to this problem based on the internal symmetry of the model, i.e. on the representation theory of quantum affine algebras. First approach is based on determinantal formulae for certain matrix elements [KBI]. For some recent results based on this method see $[\mathrm{BPZ}]$ and $[\mathrm{CP}]$. The second approach is based on form-factor formulae derived in $[\mathrm{Sm}]$. For an overview of this approach see [JM] and for the latest results see [BJMST].

The 6-vertex model on a planar simply regions can be reformulated as the theory of random stepped surfaces. A configuration of arrows in a 6-vertex model can be interpreted as a configuration of paths which can be viewed as level corves of a height function defining a stepped surface. Gibbs measure on 6-vertex configurations define a Gibbs measure on stepped surfaces.

For certain class of such Gibbs measures, random surfaces in the thermodynamical limit develop the limit shape phenomenon [Shef] also known as the acric circle phenomenon [CEP]. It means that on macroscopical scale the random surface becomes deterministic. The fluctuations remain at smaller scale and the structure of fluctuations may change depending on how singular the limit shape is at this point. The limit shape phenomenon is studied in details in dimer models [KO].

The numerical results from [AR] [SZ] show how limit shapes develop in a 6-vertex model with domain wall boundary conditions. In this paper we will focus on the limit shape phenomenon for the 6-vertex model on a planar connected simply connected regions with fixed boundary conditions. Computing the limit shape involves two steps.

Step one is the derivation of the formula for the free energy of the 6-vertex model as a function of magnetic fields. Unlike the partition function of dimer models, the free energy can not be computed explicitly. However, it can be written in terms of the solution to a linear integral equation. Many analytical properties of 
the partition function are known but scattered in the literature, see for example [Yang]|[Yang1][SY][SY1][LW] $[$ BS $][$ Nold] $[\mathrm{NK}]$. We collected most of them in the section 3 together with some new results.

Step two is to derive and solve the variational principle which determine the limit shape for given boundary conditions. The free energy of the model as a function of magnetic fields determine the functional in the variational problem. Such variational problem was first introduced for dimer models in [CKP]. The idea of using this variational problem in the 6-vertex first appeared in [Z1] where some interesting partial results were obtained for correlation functions in the bulk of the limit shape.

The structure of fluctuations near the limit shape is determined by the asymptotical behavior of correlation functions at smaller scales. We will discuss this problem for the 6-vertex model in the last section.

Finally let us mention the special case of domain wall boundary conditions. These boundary conditions first appeared in the computation of norms of Bethe vectors [Kor]. The remarkable fact about them is that the partition function of the 6-vertex model with these boundary conditions can be written as a determinant [Ize]. Another remarkable fact is that exactly these boundary conditions relate the 6-vertex model with alternating sign matrices $[\mathrm{Kup}]$. The large volume asymptotic of the partition function of the 6-vertex mode with these boundary conditions was computed in [KZ][Z].

Here is the outline of the paper. In the first two sections we recall some basic facts about the 6-vertex model, about its reformulation it in terms of height functions, and about the thermodynamical limit in the model. The third section contains the description of the free energy per site for as the function of electric fields in thermodynamical limit for periodic boundary conditions. Some asymptotical behaviors of the free energy are computed in section 4. This section is a combination of an overview and original results. In section 5 we study the asymptotical behavior of the limit shapes near the "freezing point". In the last section we discuss fluctuations.

We thank C. Evans, R. Kenyon, A. Okounkov, and S. Sheffield for interesting discussions. The work of N.R. was supported by the NSF grant DMS 0307599, by the Niels Bohr initiative at Aarhus University, by the Humboldt foundation and by the CRDF grant RUMI1-2622 The work of K.P. was supported by the NSF RTG grant and DMS 0307599.

\section{The 6-Vertex Model}

\subsection{The 6-vertex model}

First, let us fix the notation. A square $N \times M$ grid $L_{N, M}$ is a graph with 4- and 1-valent vertices embedded into $\mathbb{R}^{2}$ such that 4-valent vertices are located at points $(n, m), n=0,1, \ldots, N-1, m=0,1, \ldots, M-1$ (see Fig. 2) and 1-valent vertices are located at $(-1, m),(N, m), m=0,1, \ldots, M-1$ and at $(n,-1),(n, M-1), n=$ $0,1, \ldots, N-1$. An edge connecting two 4 -valent vertices is called an inner edge and an edge connecting a 4-valent vertex with a 1 -valent vertex is called an outer edge.

States of the 6-vertex model on $L_{N, M}$ are configurations of arrows assigned to each edge (i.e. orientations of $L_{N, M}$ ). They satisfy the ice rule: at any vertex the number of incoming arrows should be equal to the number of outgoing arrows. Six possible configurations at a vertex are shown on Fig. 1. Configurations of arrows on boundary edges are called boundary conditions. 
Each configuration of arrows on the lattice can be equivalently described as the configuration of "thin" and "thick" edges or empty and occupied edges shown on Fig. 1. There should be an even number of thick edges at each vertex as a consequence of the ice rule. The thick edges form paths. We assume that paths do not intersect (two paths may meet at an $a_{1}$-vertex). So, equivalently, configurations of the 6-vertex model can be regarded as configurations of paths satisfying the rules from Fig. 1 .

To each configuration of arrows on edges adjacent to a vertex we assign a Boltzmann weight, which we denote by the same letters. The physical meaning of a Boltzmann weight is $\exp \left(-\frac{E}{T}\right)$, where $E$ is the energy of a state and $T$ is the temperature (in the appropriate units). Thus, all numbers $a_{1}, a_{2}, b_{1}, b_{2}, c_{1}$, and $c_{2}$ should be positive.

Choosing the scale such that $T=1$, it is natural to write Boltzmann weights in the exponential form.

$$
\begin{aligned}
& a_{1}=e^{-E_{1}+H+V}, \quad a_{2}=e^{-E_{1}-H-V}, \\
& b_{1}=e^{-E_{2}+H-V}, \quad b_{2}=e^{-E_{2}-H+V}, \\
& c_{1}=e^{-E_{3}}, \quad c_{2}=e^{-E_{3}},
\end{aligned}
$$

where $E_{1}, E_{2}$, and $E_{3}$ are dimensionless interaction energies of arrows at different types of vertices, and $H$ and $V$ are dimensionless horizontal and vertical components of the magnetic field, respectively. In this interpretation arrows are spins interacting with the magnetic field. We set $c_{1}=c_{2}$ because for the types of boundary conditions we will consider the difference between the number of $c_{1}$-vertices and $c_{2}$ vertices is the same for all states and, therefore, the probability does not depend on the ratio $c_{1} / c_{2}$.

We also use the standard notation

$$
a=e^{-E_{1}}, \quad b=e^{-E_{2}}, \quad c=e^{-E_{3}} .
$$

These are the weights of the model when there is no magnetic field.

The weight of a state is the product of weights of vertices in the state. The weight of a state on $L_{N}$ (up to a constant factor) can be written in terms of energies and magnetic fields as

$$
\exp \left(-E_{1} N(a)-E_{2} N(b)-E_{3} N(c)+\frac{H}{2} N(\text { hor })+\frac{V}{2} N(\text { ver })\right)
$$

where $N(a)$ is the total number of $a$-vertices, $N(b)$ is the total number of $b$-vertices, $N(c)$ is the total number of $c$-vertices, $N($ hor $)$ is the total number of horizontal edges occupied by paths, and $N($ vert $)$ is the total number of vertical edges occupied by paths.

The partition function is the sum of weights of all states of the model

$$
Z=\sum_{\text {states }} \prod_{\text {vertices }} w(\text { vertex }),
$$

where $w$ (vertex) is one of the weights from Fig. 1 ,

Weights define the probabilistic measure on the set of states of the 6-vertex model. The probability of a state is given by the ratio of the weight of the state to the partition function of the model

$$
P(\text { state })=\frac{\prod_{\text {vertices }} w(\text { vertex })}{Z} .
$$



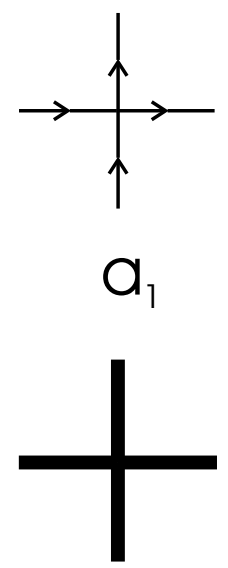

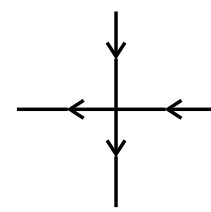

$a_{2}$

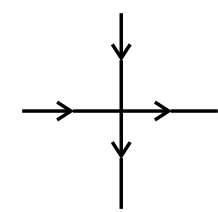

$b_{1}$

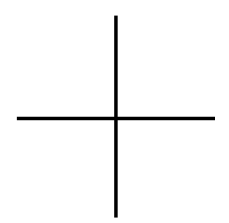

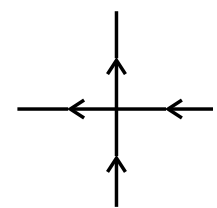

$b_{2}$

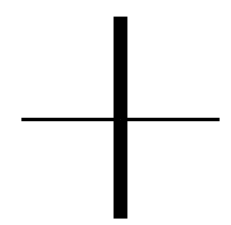

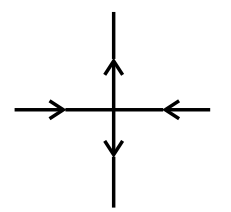

$\mathrm{C}_{1}$

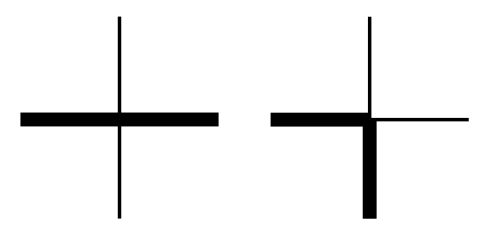

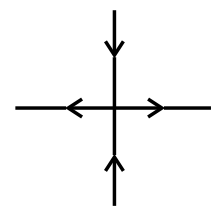

$\mathrm{C}_{2}$

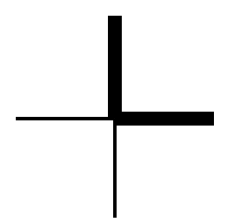

Figure 1: The 6 types of vertices and the corresponding thin and thick edges configurations

This is the Gibbs measure of the 6-vertex model.

Let us define the characteristic function of an edge $e$ as

$$
\sigma_{e}(\text { state })= \begin{cases}1, & \text { if } e \text { is occupied by a path } \\ 0, & \text { otherwise }\end{cases}
$$

A local correlation function is the expectation value of the product of such characteristic functions:

$$
\left\langle\sigma_{e_{1}} \sigma_{e_{2}} . . \sigma_{e_{n}}\right\rangle=\sum_{\text {states }} P(\text { state }) \prod_{i=1}^{n} \sigma_{e_{i}}(\text { state })
$$

\subsection{Boundary Conditions}

\subsection{1}

Let us fix arrows (or, equivalently, think edges) on outer edges of $L_{N, M}$. States in the 6-vertex with the same configurations of arrows on the boundary are called states with fixed boundary conditions. The difference between two such states can occur only at inner edges.

The space of states with fixed boundary conditions is empty unless the boundary values satisfy the ice rule: the total number of incoming arrows on the boundary edges should be equal to the total number of outgoing arrows. In the path formulation this means that the number of paths through North and West boundaries should be equal to the number paths through the South and East boundaries.

An example of such boundary conditions is the domain wall (DW) boundary conditions. For the DW boundary conditions the arrows on the boundary of the lattice are going into the lattice at the top and bottom of the lattice and are going out of the lattice at the right and left of it. A configuration of paths on a $5 \times 5$ lattice with DW boundary conditions is presented on Fig. 2 . 


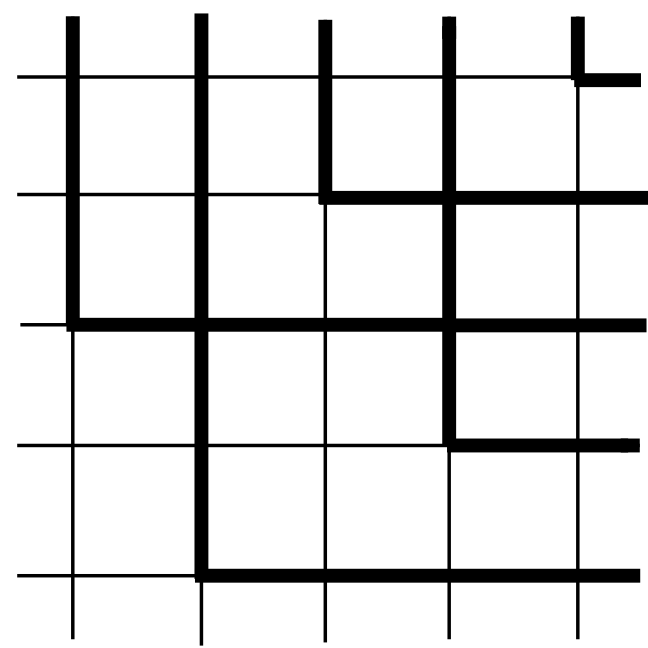

Figure 2: A possible configuration of paths on a $5 \times 5$ square grid for the DW boundary conditions

Notice that the differences

$$
n_{a}=n\left(a_{1}\right)-n\left(a_{2}\right), \quad n_{b}=n\left(b_{1}\right)-n\left(b_{2}\right), \quad n_{c}=n\left(c_{2}\right)-n\left(c_{1}\right)
$$

are the same for all configurations with given fixed boundary conditions. Here $n(x)$ is the total number of vertices of type $x=a_{i}, b_{i}, c_{i}$ in the configuration.

In particular, the partition function for fixed boundary conditions trivially depends on magnetic fields:

$$
Z\left(a_{1}, a_{2}, b_{1}, b_{2}, c_{1}, c_{2}\right)=e^{\left(H n_{b}+V n_{a}\right)}\left(\frac{c_{2}}{c_{1}}\right)^{\frac{n_{c}}{2}} Z(a, a, b, b, c, c)
$$

In this paper we will focus on the 6-vertex model with fixed boundary conditions.

\subsection{2}

Another important type of boundary conditions are the periodic boundary conditions. In this case the edges at opposite sides of $L_{N, M}$ are identified so that the configuration of arrows on the left and right boundary is the same as well as the configuration of arrows on the top and bottom boundary.

The 6-vertex model with periodic boundary conditions is an example of an "integrable" (solvable) model in statistical mechanics and has been studied extensively, see $[\overline{\mathrm{Bax}}],[\mathrm{LW}]$ and references therein. In particular, it means that the row-to-row transfer-matrix of the model can be diagonalized by the Bethe ansatz.

\subsection{The Height Function}

By outer faces we mean unit squares centered at $\left(-\frac{1}{2}, m\right),\left(N-\frac{1}{2}, m\right)$, with $m=-\frac{1}{2}, \frac{1}{2}, \ldots, M-\frac{1}{2}$ and $\left(n,-\frac{1}{2}\right),\left(n, N-\frac{1}{2}\right)$, with $n=-\frac{1}{2}, \frac{1}{2}, \ldots, N-\frac{1}{2}$. Each corner outer face has two edges in their boundary, other outer faces have three edges in their boundary. 
A height function $h$ is an integer-valued function on the faces $F_{N}$ of the grid $L_{N}$ (including the outer faces), which is

- zero at the southwest corner of the grid,

- non-decreasing when going up or right,

- if $f_{1}$ and $f_{2}$ are neighboring faces, then $\left|h\left(f_{1}\right)-h\left(f_{2}\right)\right| \leq 1$.

The boundary value of the height function is its restriction to the "outer faces". Denote the set of outer faces by $\partial F_{N}$. Given a function $h^{(0)}$ on $\partial F_{N}$ denote $\mathscr{H}\left(h^{(0)}\right)$ the space of all height functions with the boundary value $h^{(0)}$.

If we enumerate faces by the coordinates of their centers the height function can be regarded as $(N+$ 1) $\times(M+1)$ matrix with non-negative entries.

It is clear that there is a bijection between states of the 6-vertex model with fixed boundary conditions and height functions with fixed boundary values.

Indeed, given a height function consider its "level curves", i.e. paths on the grid $L_{N}$, where the height function changes its value by 1 , see Fig. 3. Clearly, this defines a state for the 6-vertex model on $L_{N}$ with boundary conditions determined by the boundary values of the height function.

On the other hand, given a state in the 6-vertex model, consider the corresponding configuration of paths. It is clear that there is a unique height function whose level curves are these paths and which satisfies the condition $h=0$ at the southwest corner.

It is clear that this correspondence is a bijection.

There is a natural partial order on the set of height functions with given boundary values. One function is bigger then the other if it is entirely above the other. There exist the minimum $h_{\min }$ and the maximum $h_{\max }$ height functions such that $h_{\min } \leq h \leq h_{\max }$ for all height functions $h$.

Thus, we can consider the 6-vertex model as a theory of fluctuating discrete surfaces constrained between $h_{\max }$ and $h_{\min }$. Each surface occurs with probability given by the Boltzmann weights of the 6-vertex model.

\subsection{The inhomogeneous 6-vertex model and volume weights}

In the inhomogeneous 6-vertex model the Boltzmann weights depend on the edge. Thus, we have $6 N^{2}$ parameters $a_{i}(m, n), b_{i}(m, n)$, and $c_{i}(m, n)$.

Let us assume that the inhomogeneity is only in magnetic fields, i.e. weights $a, b$, and $c$ do not change from edge to edge, but the magnetic fields $H(e), V(e)$ do.

Let $\{P\}$ be the collection of paths corresponding to a state in the 6-vertex model and $\{h(f)\}$ be the corresponding height function.

Proposition 2.1. Let us assign weights $s(e)$ to the edges of the lattice and 1 to the outer edges, then

$$
\prod_{f} q_{f}^{h(f)}=\prod_{P} \prod_{e \in P} s(e)
$$




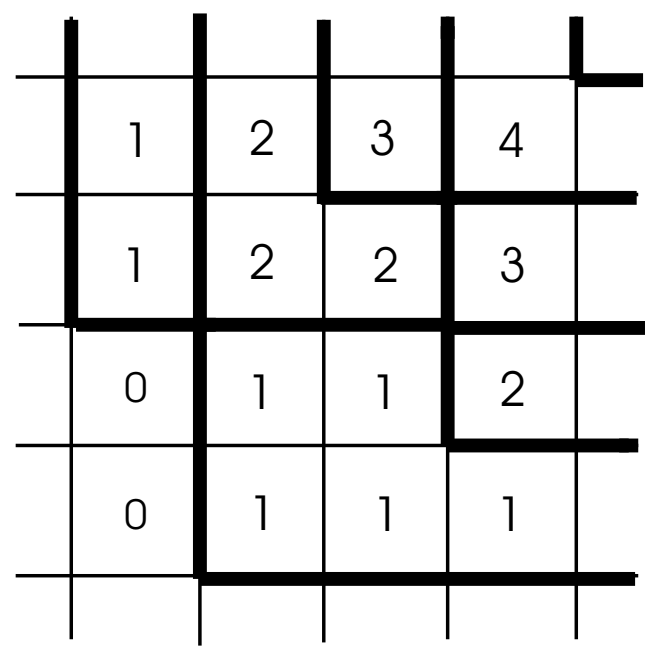

Figure 3: Values of the height function for the configuration of paths given on figure 2.

where $q_{f}=s\left(e_{1}\right) s\left(e_{2}\right) s\left(e_{3}\right)^{-1} s\left(e_{4}\right)^{-1}$ for inner faces, $q_{f}=s(e)^{\varepsilon(e)}$ for the outer face $f$ adjacent to the edge $e, q_{f}=1$ for outer faces not adjacent to any edge of the lattice (corner faces) with $\varepsilon(e)=1$ for edges at the upper and right sides of the boundary, $\varepsilon(e)=-1$ for edges at the lower and left sides of the boundary.

The proof is an elementary exercise.

Let $s(e)=\exp (H(e)$ for horizontal edges and $s(e)=\exp (V(e))$ for vertical edges. Then the probability of the state with the height function $h$ in such a model is

$$
P_{q}(\text { state })=\frac{\prod_{f} q_{f}^{h(f)} \prod_{\text {vertices }} w_{0}(\text { vertex })}{Z}
$$

If $H(n, m)=H+a m$ and $V(n, m)=V+b n$, the weights $q_{f}$ are the same for all faces $q_{f}=\exp (a+b)$ inside the lattice and the probability is given by

$$
P(\text { state })=\frac{q^{\mathrm{vol}(h)} \prod_{\mathrm{vertices}} w(\mathrm{vertex})}{Z},
$$

where $w$ (vertex) are the Boltzmann weights with constant magnetic fields and

$$
\operatorname{vol}(h)=\sum_{f \in L_{N}} h(f)
$$

is the volume "under" the height function $h$. 


\section{The thermodynamic limit}

\subsection{Stabilizing sequence of fixed boundary conditions}

\subsection{1}

Let $a=M / N$. We place the grid $L_{N, M}$ inside of the rectangle $D=\{(x, y) \mid 0 \leq x \leq 1,0 \leq y \leq a\}$ so that the vertices of the grid are the points with coordinates $\left(\frac{n}{N+1}, \frac{m}{N+1}\right)$ where $n=1, \ldots, N, m=1, \ldots, M$.

We recall that a height function is a monotonic integer-valued function on the faces of the grid, which satisfies Lipshitz condition (it changes at most by 1 on any two adjacent faces). The height function can be regarded as a function on the centers of the faces of the grid, i.e on $(n-1 / 2, m-1 / 2)$, where $n=$ $0, \ldots, N+1, m=0,1, \ldots, M+1$. The points $\left(\frac{1}{2}, m-\frac{1}{2}\right),\left(N+\frac{1}{2}, m-\frac{1}{2}\right),\left(n-\frac{1}{2}, \frac{1}{2}\right)$, and $\left(n-\frac{1}{2}, M+\frac{1}{2}\right)$, where $n=0, \ldots, N+1, m=0, \ldots, M+1$ correspond to the "outer" faces of $L_{N, M}$.

We introduce the normalized height function as a piecewise linear function on the unit square with the value

$$
h_{N}^{\mathrm{norm}}(x, y)=\frac{1}{N} h_{N}(n, m) .
$$

for $\frac{n}{N+1} \leq x \leq \frac{n+1}{N+1}$ and $\frac{m}{N+1} \leq y \leq \frac{m+1}{N+1}$. Here $h_{N}(n, m)$ is a height function on $L_{N, M}$. Normalized height functions are nondecreasing in $x$ and $y$ directions and they satisfy:

$$
h(x, y)-h\left(x^{\prime}, y^{\prime}\right) \leq x-x^{\prime}+y-y^{\prime} .
$$

if $x \geq x^{\prime}$ and $y \geq y^{\prime}$.

The boundary value of the normalized height function defines a piece-wise constant monotonic function on each side of the region which changes by $\pm 1 / N$ or do not change between two neighboring boundary sites.

Denote the space of such normalized height functions with the boundary value $h_{0}$ by $L_{N, M}\left(h_{0}\right)$.

There is a natural partial ordering on the set of all normalized height functions with given boundary values descending from the partial order on height functions: $h_{1} \geq h_{2}$ if $h_{1}(x) \geq h_{2}(x)$ for all $x \in D$. We define the operations

$$
h_{1} \vee h_{2}=\min _{x \in D}\left(h_{1}(x), h_{2}(x)\right), h_{1} \wedge h_{2}=\max _{x \in D}\left(h_{1}(x), h_{2}(x)\right),
$$

It is clear that

$$
h_{1} \wedge h_{2} \geq h_{1}, h_{2} \geq h_{1} \vee h_{2}
$$

It is also clear that in this partial order there is unique minimal and unique maximal height functions, which we denote by $h_{N}^{\min }$ and $h_{N}^{\max }$, respectively.

A sequence of stabilizing fixed boundary conditions is a sequence of functions $h_{0}^{(N)}$ which are boundary values of a normalized height function, and which converges ??? as $N \rightarrow \infty$ to a function $\phi_{0}$ on the boundary of $D$ which is non-decreasing along $x$ and $y$ direction, $\phi(0,0)=0$, and satisfies the condition:

$$
\phi(x, y)-\phi\left(x^{\prime}, y^{\prime}\right) \leq x-x^{\prime}+y-y^{\prime}
$$


We will call the function $\phi_{0}$ the boundary condition for the domain $D$ (or simply the boundary condition). Any function on $D$ which satisfies (3.2), which is non-decreasing in $x$ and $y$ directions, and coinside with $\phi_{0}$ at the boundary is called a height function on $D$ with boundary condition $\phi_{0}$.

Among all possible boundary conditions $\phi_{0}$ we will distinguish piecewise linear boundary conditions with the slope 0 or 1 along the coordinate axes. We will call these boundary values critical. It is clear that any boundary conditions can be approximated critical boundary conditions.

\subsection{The thermodynamic limit}

If $q$ is fixed and is not equal to 1 in the large volume limit, the system will be in a neighborhood of the minimal height function for $q<1$ and in the neighborhood of the maximal height function for $q>1$. One should expect that the partition function and local correlation functions will have finite limit.

When $q=\exp \left(\frac{\lambda}{N}\right)$ for some $\lambda$, one should expect the existence of the limit shape. We will study this limit in Section 7 .

\subsection{Gibbs measures with fixed slope}

Definition 3.1. The Gibbs measure of the 6-vertex model on an infinite lattice has the slope $(h, v)$ if

$$
\lim _{k \rightarrow \infty}<\frac{h(n+k, m)-h(n, m)}{k}>=h
$$

and

$$
\lim _{k \rightarrow \infty}<\frac{h(n, m+k)-h(n, m)}{k}>=v
$$

It is clear from the definition of the height function that the slope should satisfy conditions $0 \leq h, v \leq 1$.

The slope is simply the average number of horizontal and vertical edges occupied by paths per length.

The important corollary of the result of [Shef] is that, when a gradient Gibbs measure satisfies certain convexity conditions, there exists a unique translationally invariant measure. This implies that for the 6vertex model one should expect the uniqueness of such a measure for the generic slope.

Translationally invariant measures can be obtained by taking the thermodynamic limit of the 6-vertex model with magnetic fields on a torus. Then the slope is the Legendre conjugate to magnetic fields.

$$
h=\lim _{N, M \rightarrow \infty}\left\langle\sigma_{e}\right\rangle=\lim _{N, M \rightarrow \infty}\left\langle\frac{N(\text { hor })}{N}\right\rangle=\lim _{N, M \rightarrow \infty} \frac{1}{2 N M} \frac{\partial \log Z}{\partial H}+\frac{1}{2}
$$

for a horizontal $e$ and

$$
v=\lim _{N . M \rightarrow \infty}\left\langle\sigma_{e}\right\rangle=\lim _{N, M \rightarrow \infty}\left\langle\frac{N(\text { ver })}{N}\right\rangle=\lim _{N, M \rightarrow \infty} \frac{1}{2 N M} \frac{\partial \log Z}{\partial V}+\frac{1}{2}
$$

for a vertical $e$. 


\section{The thermodynamic limit of the 6-vertex model for the periodic boundary conditions}

The free energy per site in the thermodynamic limit is

$$
f=-\lim _{N, M \rightarrow \infty} \frac{\log \left(Z_{N, M}\right)}{N M}
$$

where $Z_{N, M}$ is the partition function with the periodic boundary conditions on the rectangular grid $L_{N, M}$. It is a function of the Boltzmann weights and magnetic fields.

Remark 1. Normally, it is expected that the free energy is not identically zero. Physically, this means that the "excitations" have the characteristic length which is much smaller than the characteristic length of the system. In some cases the free energy is identically zero, then one expects that a typical excitation will be comparable with the size of the system.

For generic $H$ and $V$ the 6-vertex model in the thermodynamic limit has the translationally invariant Gibbs measure with the slope $(h, v)$ :

$$
h=-\frac{1}{2} \frac{\partial f}{\partial H}+\frac{1}{2}, \quad v=-\frac{1}{2} \frac{\partial f}{\partial V}+\frac{1}{2} .
$$

The parameter

$$
\Delta=\frac{a^{2}+b^{2}-c^{2}}{2 a b} .
$$

defines many characteristics of the 6-vertex model in the thermodynamic limit.

\subsection{The phase diagram for $\Delta>1$}

The weights $a, b$, and $c$ in this region satisfy one of the two inequalities, either $a>b+c$ or $b>a+c$. If $a>b+c$, the Boltzmann weights $a, b$, and $c$ can be parameterized as

$$
a=r \sinh (\lambda+\eta), b=r \sinh (\lambda), c=r \sinh (\eta)
$$

with $\lambda, \eta>0$.

If $a+c<b$, the Boltzmann weights can be parameterized as

$$
a=r \sinh (\lambda-\eta), b=r \sinh (\lambda), c=r \sinh (\eta)
$$

with $0<\eta<\lambda$.

For both of these parametrization of weights $\Delta=\cosh (\eta)$.

The phase diagram of the model for $a>b+c$ (and, therefore, $a>b$ ) is shown on Fig. 5 and for $b>a+c$ (and, therefore, $a<b$ ) on Fig. 6 .

When magnetic fields $(H, V)$ are in one of the regions $A_{i}, B_{i}$ of the phase diagram, the system in the thermodynamic limit has the translationally invariant Gibbs measure supported on the corresponding frozen 
configuration. There are four frozen configurations $A_{1}, A_{2}, B_{1}$, and $B_{2}$, shown on Fig. 4 . For a finite but large grid the probability of any other state is at most of order $\exp (-\alpha N)$ for some positive $\alpha$.

Local correlation functions are given by the value of the corresponding observable on the frozen state

$$
\lim _{N \rightarrow \infty}\left\langle\sigma_{e_{1}} \ldots \sigma_{e_{n}}\right\rangle_{N}=\sigma_{e_{1}}(S) \ldots \sigma_{e_{n}}(S)
$$

where $S$ is the one of the ferromagnetic states $A_{i}, B_{i}$.

The frozen regions in the $(H, V)$-plane are described by the set of inequalities. The boundaries of these regions can be derived by analyzing the next to the largest eigenvalue of the row-to-row transfer matrix. The description is separated into two cases: $a>b+c$ and $b>a+c$. Notice that $a \neq b$ since $\Delta>1$.

- $a>b+c$, see Fig. 5 ,

$$
\begin{aligned}
& A_{1} \text {-region: } \quad V+H \geq 0, \quad \cosh (2 H) \leq \Delta, \\
& \quad\left(e^{2 H}-b / a\right)\left(e^{2 V}-b / a\right) \geq(c / a)^{2}, \quad e^{2 H}>b / a, \quad \cosh (2 H)>\Delta, \\
& A_{2} \text {-region: } \quad V+H \leq 0, \quad \cosh (2 H) \leq \Delta, \\
& \quad\left(e^{-2 H}-b / a\right)\left(e^{-2 V}-b / a\right) \geq(c / a)^{2}, \quad e^{-2 H}>b / a, \quad \cosh (2 H)>\Delta, \\
& B_{1} \text {-region: } \quad\left(e^{2 H}-a / b\right)\left(e^{-2 V}-a / b\right) \geq(c / b)^{2}, \quad e^{2 H}>a / b, \\
& B_{2} \text {-region: } \quad\left(e^{-2 H}-a / b\right)\left(e^{2 V}-a / b\right) \geq(c / b)^{2}, \quad e^{-2 H}>a / b .
\end{aligned}
$$

- $b>a+c$, see Fig. 6 ,

$$
\begin{aligned}
& A_{1} \text {-region: } \quad\left(e^{2 H}-b / a\right)\left(e^{2 V}-b / a\right) \geq(c / a)^{2}, \quad e^{2 H}>b / a \text {; } \\
& A_{2} \text {-region: } \quad\left(e^{-2 H}-b / a\right)\left(e^{-2 V}-b / a\right) \geq(c / a)^{2}, \quad e^{-2 H}>b / a \text {; } \\
& B_{1} \text {-region: } \quad V-H \geq 0, \quad \cosh (2 H) \leq \Delta \text {, } \\
& \left(e^{2 H}-a / b\right)\left(e^{-2 V}-a / b\right) \geq(c / b)^{2}, \quad e^{2 H}>a / b, \quad \cosh (2 H)>\Delta, \\
& B_{2} \text {-region: } \quad V-H \leq 0, \quad \cosh (2 H) \leq \Delta \text {, } \\
& \left(e^{-2 H}-a / b\right)\left(e^{2 V}-a / b\right) \geq(c / b)^{2}, \quad e^{-2 H}>a / b, \quad \cosh (2 H)>\Delta .
\end{aligned}
$$

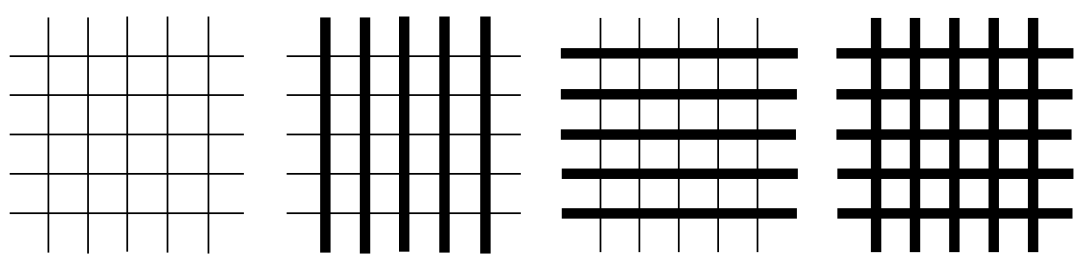

Figure 4: Four frozen configurations of the ferromagnetic phase 


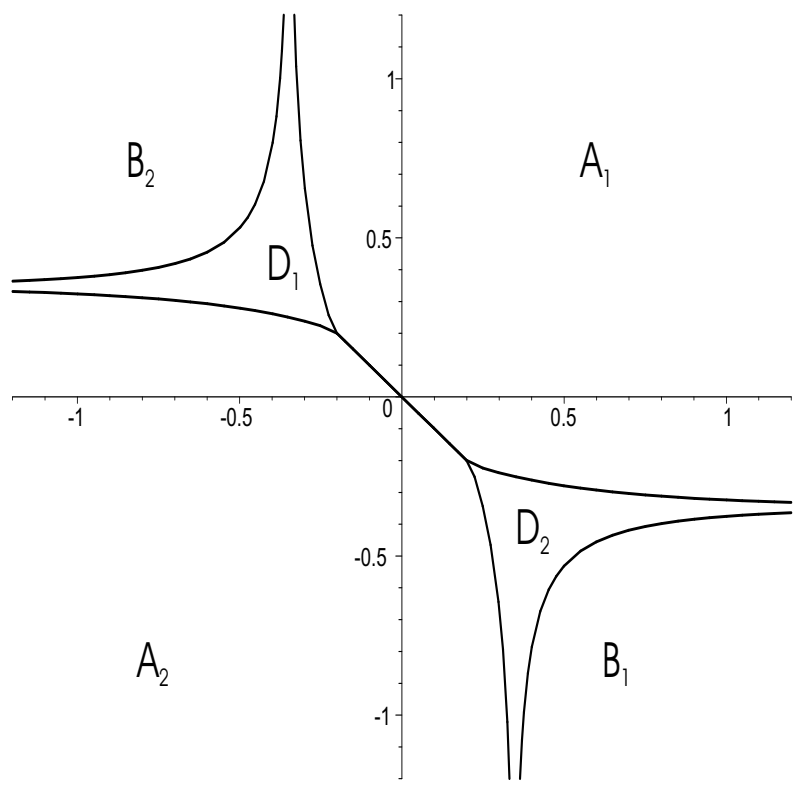

Figure 5: The phase diagram in the $(H, V)$-plane for $a=2, b=1$, and $c=0.8$

The free energy is a linear function in $H$ and $V$ in the four frozen regions:

$$
\begin{array}{ll}
f=-\ln a-H-V & \text { in } \quad A_{1}, \\
f=-\ln b+H-V & \text { in } \quad B_{2}, \\
f=-\ln a+H+V & \text { in } \quad A_{2}, \\
f=-\ln b-H+V & \text { in } \quad B_{1} .
\end{array}
$$

If $(H, V)$ is a region $D_{1}$ or $D_{2}$ the corresponding translationally invariant Gibbs measure has the slope $(h, v)$ given by (4.1). In this phase the system is disordered, which means that local correlation functions decay as a power of the distance $d\left(e_{i}, e_{j}\right)$ between $e_{i}$ and $e_{j}$ when $d\left(e_{i}, e_{j}\right) \rightarrow \infty$.

In the regions $D_{1}$ and $D_{2}$ the free energy is given by [SY]:

$$
\begin{aligned}
f(H, V)= & \min _{\alpha}\left\{\min _{\alpha}\left\{E_{1}-H-(1-2 \alpha) V-\frac{1}{2 \pi i} \int_{C} \ln \left(\frac{b}{a}-\frac{c^{2}}{a b-a^{2} z}\right) \rho(z) d z\right\}\right. \\
& \left.\min _{\alpha}\left\{E_{2}+H-(1-2 \alpha) V-\frac{1}{2 \pi i} \int_{C} \ln \left(\frac{a^{2}-c^{2}}{a b}+\frac{c^{2}}{a b-a^{2} z}\right) \rho(z) d z\right\}\right\},
\end{aligned}
$$

where $\rho(z)$ can be found from the integral equation

$$
\rho(z)=\frac{1}{z}+\frac{1}{2 \pi i} \int_{C} \frac{\rho(w)}{z-z_{2}(w)} d w-\frac{1}{2 \pi i} \int_{C} \frac{\rho(w)}{z-z_{1}(w)} d w
$$




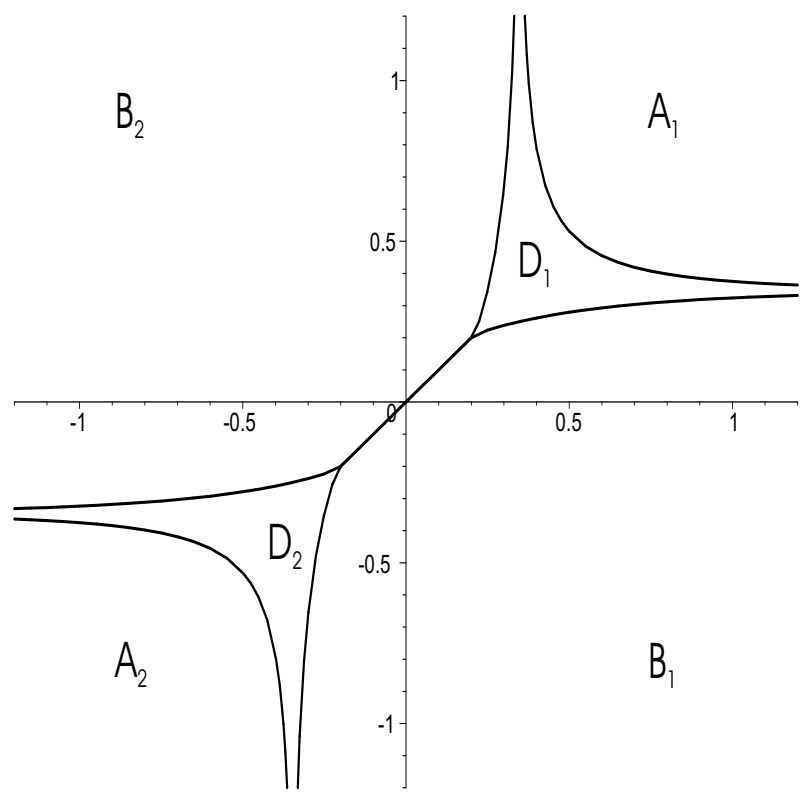

Figure 6: The phase diagram in the $(H, V)$-plane for $a=1, b=2$, and $c=0.8$

in which

$$
z_{1}(w)=\frac{1}{2 \Delta-w}, \quad z_{2}(w)=-\frac{1}{w}+2 \Delta .
$$

$\rho(z)$ satisfies the following normalization condition:

$$
\alpha=\frac{1}{2 \pi i} \int_{C} \rho(z) d z .
$$

The contour of integration $C$ (in the complex $z$-plane) is symmetric with respect to the conjugation $z \rightarrow \bar{z}$, is dependent on $H$ (see Appendix $(\mathrm{B}$ ) and is defined by the condition that the form $\rho(z) d z$ has purely imaginary values on the vectors tangent to $C$ :

$$
\left.\operatorname{Re}(\rho(z) d z)\right|_{z \in C}=0
$$

The formula (4.5) for the free energy follows from the Bethe Ansatz diagonalization of the row-torow transfer-matrix. Its derivation is outlined in Appendix B It relies on a number of conjectures that are supported by numerical and analytical evidence and in physics are taken for granted. However, there is no rigorous proof.

There are two points where three phases coexist (two frozen and one disordered phase). These points are called tricritical. The angle $\theta$ between the boundaries of $D_{1}$ (or $D_{2}$ ) at a tricritical point is given by

$$
\cos (\theta)=\frac{c^{2}}{c^{2}+2 \min (a, b)^{2}\left(\Delta^{2}-1\right)} .
$$


The existence of such points makes the 6-vertex model (and its degeneration known as the 5-vertex model $[$ HWKK $]$ ) remarkably different from dimer models [KOS] where generic singularities in the phase diagram are cusps. Physically, the existence of singular points where two curves meet at the finite angle manifests the presence of interaction in the 6-vertex model.

Notice that when $\Delta=1$ the phase diagram of the model has a cusp at the point $H=V=0$. This is the transitional point between the region $\Delta>1$ and the region $|\Delta|<1$ which is described below.

\subsection{The phase diagram $|\Delta|<1$}

In this case, the Boltzmann weights have a convenient parameterization by trigonometric functions. When $1 \geq \Delta \geq 1$

$$
a=r \sin (\lambda-\gamma), b=r \sin (\lambda), c=r \sin (\gamma),
$$

where $0 \leq \gamma \leq \pi / 2, \gamma \leq \lambda \leq \pi$, and $\Delta=\cos \gamma$.

When $0 \geq \Delta \geq-1$

$$
a=r \sin (\gamma-\lambda), b=r \sin (\lambda), c=r \sin (\gamma),
$$

where $0 \leq \gamma \leq \pi / 2, \pi-\gamma \leq \lambda \leq \pi$, and $\Delta=-\cos \gamma$.

The phase diagram of the 6-vertex model with $|\Delta|<1$ is shown on Fig. 7. The phases $A_{i}, B_{i}$ are frozen and identical to the frozen phases for $\Delta>1$. The phase $D$ is disordered. For magnetic fields $(H, V)$ the Gibbs measure is translationally invariant with the slope $(h, v)=\left(\frac{\partial f(H, V)}{\partial H}, \frac{\partial f(H, V)}{\partial V}\right)$.

The frozen phases can be described by the following inequalities:

$$
\begin{array}{lll}
A_{1} \text {-region: } & \left(e^{2 H}-b / a\right)\left(e^{2 V}-b / a\right) \geq(c / a)^{2}, & e^{2 H}>b / a, \\
A_{2} \text {-region: } & \left(e^{-2 H}-b / a\right)\left(e^{-2 V}-b / a\right) \geq(c / a)^{2}, & e^{-2 H}>b / a, \\
B_{1} \text {-region: } & \left(e^{2 H}-a / b\right)\left(e^{-2 V}-a / b\right) \geq(c / b)^{2}, & e^{2 H}>a / b, \\
B_{2} \text {-region: } & \left(e^{-2 H}-a / b\right)\left(e^{2 V}-a / b\right) \geq(c / b)^{2}, & e^{-2 H}>a / b .
\end{array}
$$

The free energy function in the frozen regions is still given by the formulae (4.5). The first derivatives of the free energy are continuous at the boundary of frozen phases, The second derivative is continuous in the tangent direction at the boundary of frozen phases and is singular in the normal direction.

It is smooth in the disordered region where it is given by (4.5) which, as in case $\Delta>1$ involves a solution to the integral equation (4.6). The contour of integration in (4.6) is closed for zero magnetic fields and, therefore, the equation (4.6) can be solved explicitly by the Fourier transformation [Bax] .

The 6-vertex Gibbs measure with zero magnetic fields converges in the thermodynamic limit to the superposition of translationally invariant Gibbs measures with the slope $(1 / 2,1 / 2)$. There are two such measures. They correspond to the double degeneracy of the largest eigenvalue of the row-to-row transfermatrix $[\mathrm{Bax}]$.

There is a very interesting relationship between the 6-vertex model in zero magnetic fields and the highest weight representation theory of the corresponding quantum affine algebra. The double degeneracy 


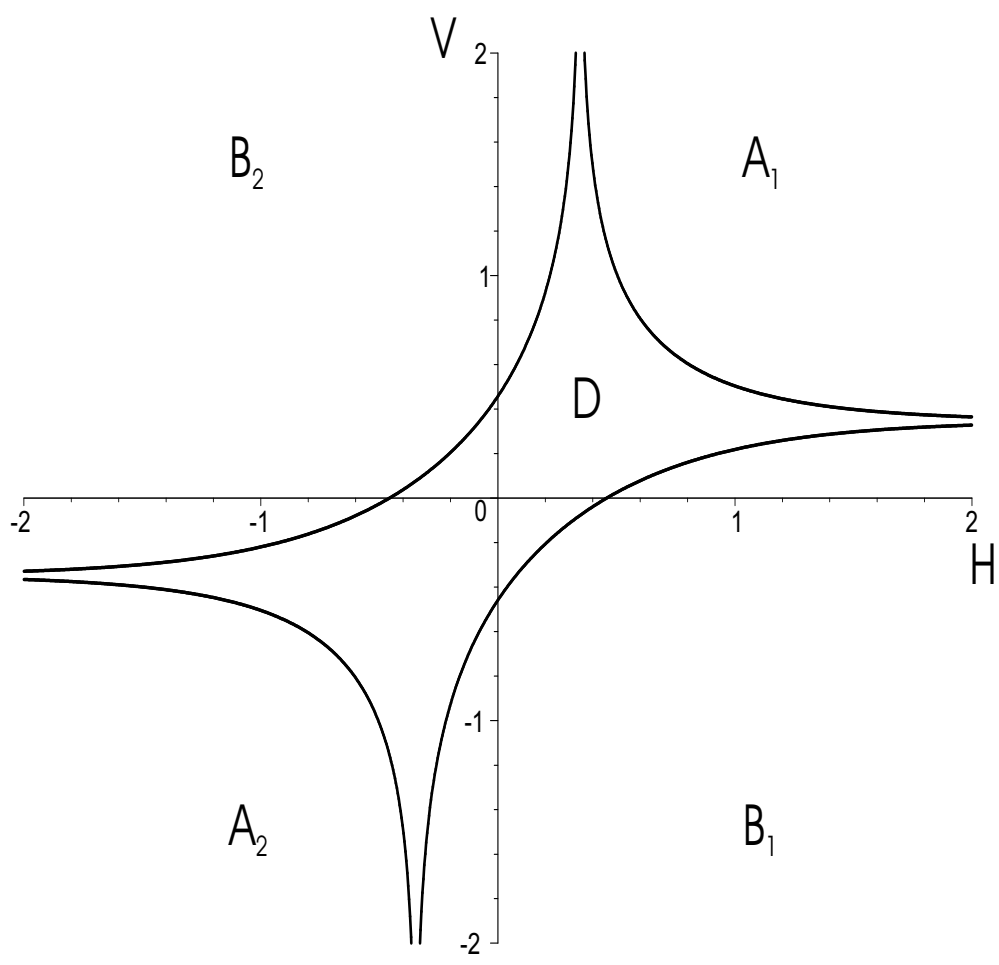

Figure 7: The phase diagram in the $(H, V)$-plane for $a=1, b=2$, and $c=2$

of the Gibbs measure with the slope $(1 / 2,1 / 2)$ corresponds to the fact that there are two integrable irreducible representations of $\widehat{s l_{1}}$ at level one. Correlation functions in this case can be computed using $q$-vertex operators [JM]. For latest developments see [BJMST].

\subsection{The phase diagram $\Delta<-1$}

\subsubsection{The phase diagram}

The Boltzmann weights for these values of $\Delta$ can be conveniently parameterized as

$$
a=r \sinh (\eta-\lambda), b=r \sinh (\lambda), c=r \sinh (\eta),
$$

where $0<\lambda<\eta$ and $\Delta=-\cosh \eta$.

The Gibbs measure in thermodynamic limit depends on the value of magnetic fields. The phase diagram in this case is shown on Fig. 8 for $b / a>1$. In the parameterization (4.8) this correspond to $0<\lambda<\eta / 2$. When $\eta / 2<\lambda<\eta$ the 4-tentacled "amoeba" is tilted in the opposite direction as on Fig. 5. When $(H, V)$ is in one of the $A_{i}, B_{i}$ regions in the phase diagram the Gibbs measure is supported on the corresponding frozen configuration, see Fig. 4 


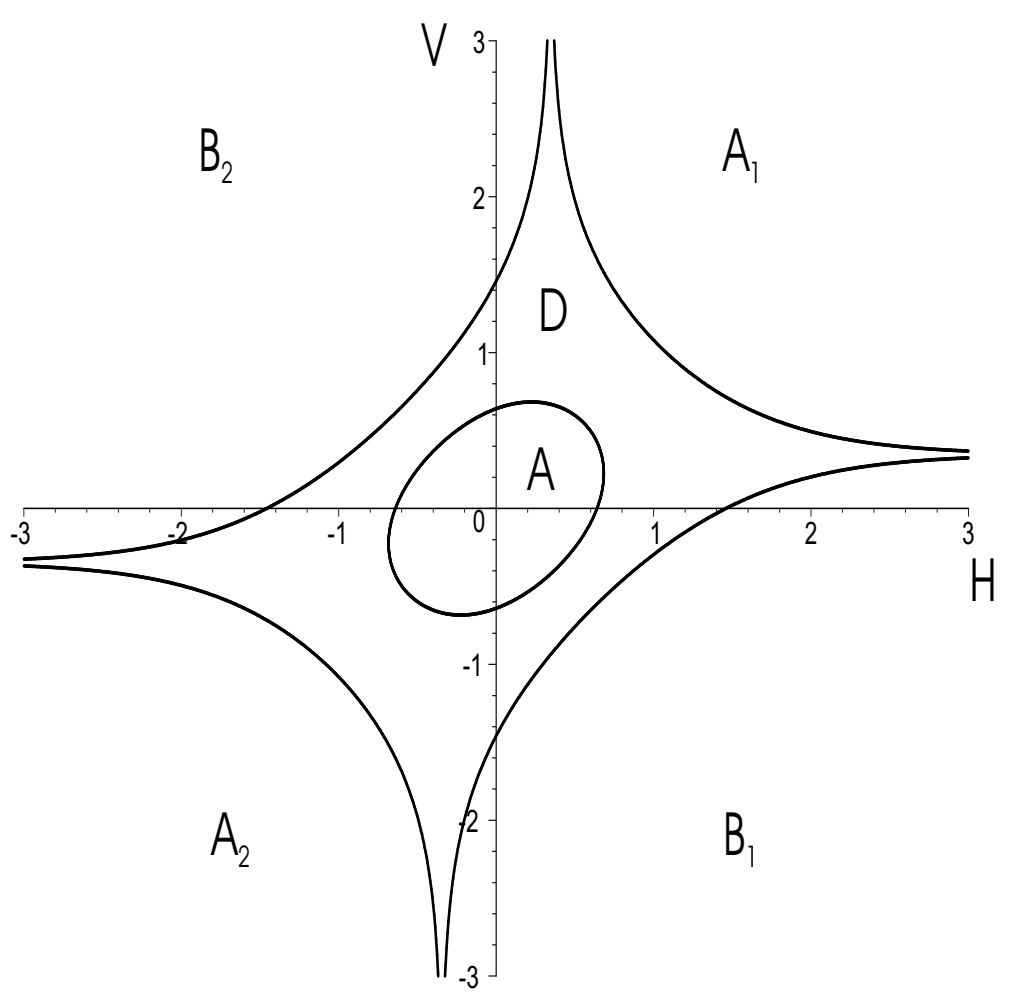

Figure 8: The phase diagram in the $(H, V)$-plane for $a=1, b=2$, and $c=6$

The $A_{i}, B_{i}$ regions on the phase diagram are defined by inequalities (4.7). The free energy in these regions is linear and is given by (4.5).

If $(H, V)$ is in the region $D$, the Gibbs measure is the translationally invariant measure with the slope $(h, v)$ determined by (4.1). The free energy in this case is determined by solutions to the linear integral equation (4.6) and is given by the formula (4.5).

If $(H, V)$ is in the region $A$, the Gibbs measure is the superposition of two Gibbs measures with the slope $(1 / 2,1 / 2)$. In the limit $\Delta \rightarrow-\infty$ these two measures degenerate to two measures supported on configurations $C_{1}, C_{2}$, respectively, shown on Fig. 9 . For a finite $\Delta$ the support of these measures consists of configurations which differ from $C_{1}$ and $C_{2}$ in finitely many places on the lattice.

We notice that any two configurations lying in the support of each of these Gibbs measures can be obtained from $C_{1}$ or $C_{2}$ via flipping the path at a vertex "up" or "down" as it is shown on Fig. 10 finitely many times. It is also clear that it takes infinitely many flips to go from $C_{1}$ to $C_{2}$.

The 6-vertex model in the phase $A$ is disordered and is also noncritical.

Here non-criticality means that the local correlation function $\left\langle\sigma_{e_{i}} \sigma_{e_{j}}\right\rangle$ decays as $\exp \left(-\alpha d\left(e_{i}, e_{j}\right)\right)$ with some positive $\alpha$ as the distance $d\left(e_{i}, e_{j}\right)$ between $e_{i}$ and $e_{j}$ increases to infinity.

The free energy in the $A$-region can be explicitly computed by solving the equation (4.6) via the Fourier 
transform $[\mathrm{Bax}]$.

\subsubsection{The antiferromagnetic region}

This boundary of the antiferromagnetic region $A$ can be derived similarly to the boundaries of the ferromagnetic regions $A_{i}$ and $B_{i}$ by analyzing next to the largest eigenvalue of the row-to-row transfer-matrix. The difference is that for the region $A$ the largest eigenvalue will correspond to $n=N / 2$ and to compute it we should use the solution to the integral equation (4.6) in the case when the contour of integration is closed.

This computation was done in [SY], [LW]. The result is a simple closed curve, which can be described parameterically as

$$
H(s)=\Xi(s), \quad V(s)=\Xi\left(\eta-\theta_{0}+s\right),
$$

where

$$
\begin{gathered}
\Xi(\varphi)=\cosh ^{-1}\left(\frac{1}{\operatorname{dn}\left(\frac{K}{\pi} \varphi \mid 1-v\right)}\right), \\
|s| \leq 2 \eta
\end{gathered}
$$

and

$$
e^{\theta_{0}}=\frac{1+\max (b / a, a / b) e^{\eta}}{\max (b / a, a / b)+e^{\eta}} .
$$

The parameter $v$ is defined by the equation $\eta K(v)=\pi K^{\prime}(v)$, where

$$
K(v)=\int_{0}^{\pi / 2}\left(1-v \sin ^{2}(\theta)\right)^{-1 / 2} d \theta \quad K^{\prime}(v)=\int_{0}^{\pi / 2}\left(1-(1-v) \sin ^{2}(\theta)\right)^{-1 / 2} d \theta
$$

The curve is invariant with respect to the reflections $(H, V) \rightarrow(-H,-V)$ and $(H, V) \rightarrow(V, H)$ since the function $\Xi$ satisfies the identities

$$
\Xi(\varphi)=-\Xi(-\varphi), \quad \Xi(\eta-\varphi)=\Xi(\eta+\varphi) .
$$

This function is also $4 \eta$-periodic: $\Xi(4 \eta+\varphi)=\Xi(\varphi)$.
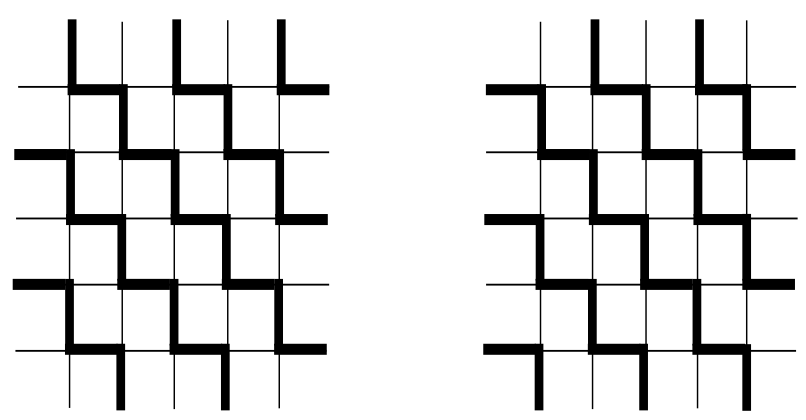

Figure 9: Two most probable configurations in the antiferromagnetic phase. 


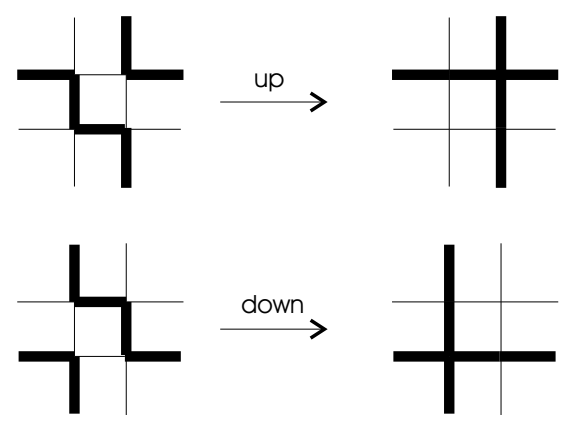

Figure 10: The elementary up and down fluctuations in the antiferromagnetic phase.

Proposition 4.1. The boundary of the antiferromagnetic region is a real algebraic curve in $e^{H}$ and $e^{V}$ given by

$$
\begin{aligned}
& \left(\left(1-v \cosh ^{2} V_{0}\right) \cosh ^{2} H+\sinh ^{2} V_{0}-(1-v) \cosh V_{0} \cosh H \cosh V\right)^{2}= \\
& \left(1-v \cosh ^{2} V_{0}\right) \sinh ^{2} V_{0} \cosh ^{2} V \sinh ^{2} H\left(1-v \cosh ^{2} H\right),
\end{aligned}
$$

where $V_{0}$ is the positive value of $V$ on the curve when $H=0$. Notice that $v$ depends on the Boltzmann weights $a, b, c$ only through $\eta$.

Proof. The parametric description of the boundary curve implies that

$$
\operatorname{dn}\left(\frac{K}{\pi} s \mid 1-v\right)=\frac{1}{\cosh H} \quad \operatorname{dn}\left(\frac{K}{\pi}\left(\eta-\theta_{0}+s\right) \mid 1-v\right)=\frac{1}{\cosh V}
$$

The addition formula for the Jacobi elliptic function dn [AS]

$$
\operatorname{dn}(u+v)=\frac{\operatorname{dn} u \operatorname{dn} v-(1-m) \operatorname{sn} u \operatorname{cn} u \operatorname{sn} v \operatorname{cn} v}{1-(1-m) \operatorname{sn}^{2} u \mathrm{sn}^{2} v} .
$$

can be used to express $\operatorname{dn} u$ and $\operatorname{dn} v$ for $u=K\left(\eta-\theta_{0}\right) / \pi$ and $v=K s / \pi$ in terms of $N$ and $V$. Using elementary identities for the elliptic functions, we obtain

$$
\operatorname{sn}^{2} u \operatorname{cn}^{2} u\left(\operatorname{dn}^{2} v-v\right)\left(1-\operatorname{dn}^{2} v\right)=\left(\operatorname{dn} u \operatorname{dn} v-\operatorname{dn}(u+v)\left(\operatorname{cn}^{2} u+\operatorname{sn}^{2} u \operatorname{dn}^{2} v\right)\right)^{2}
$$

For $u=K\left(\eta-\theta_{0}\right) / \pi$ and $v=K s / \pi$ this identity turns into

$$
\left(\operatorname{cn}^{2} u \cosh ^{2} H+\operatorname{sn}^{2} u-\operatorname{dn} u \cosh H \cosh V\right)^{2}=\operatorname{cn}^{2} u \operatorname{sn}^{2} u \cosh ^{2} V\left(\cosh ^{2} H-1\right)\left(1-m \cosh ^{2} H\right) .
$$

Denote $\operatorname{dn}\left(\frac{K}{\pi}\left(\eta-\theta_{0}\right) \mid 1-m\right)=1 / \cosh V_{0}$, then this identity becomes (4.9). 


\section{Some asymptotics of the free energy}

\subsection{The scaling near the boundary of the $D$-region}

Here we study the asymptotics of the free energy of the 6-vertex model as the point $(H, V)$ inside a disordered region approaches its boundary on the phase diagram of the model.

Let us consider the interface between the disordered region $D\left(D_{2}\right.$ for $\left.\Delta>1\right)$ and the $A_{1}$-region, see Fig. 7. It is given by $g(H, V)=0$, where

$$
g(H, V)=\ln \left(b / a+\frac{c^{2} / a^{2}}{e^{2 H}-b / a}\right)-2 V .
$$

Let $\vec{H}_{0}=\left(H_{0}, V_{0}\right)$ be a regular point on the interface, i.e. the interface can be parameterized by real analytic functions in its neighborhood, then $g\left(H_{0}, V_{0}\right)=0$.

We denote the normal vector to the interface at $\vec{H}_{0}$ by $\vec{n}$ and the tangent vector by $\vec{\tau}$. A point $\vec{H}=(H, V)$ in the vicinity of $\vec{H}_{0}$ can be represented as $\vec{H}(r, s, t)=\vec{H}_{0}+r^{2} s \vec{n}+r t \vec{\tau}$, where $s$ and $t$ are local coordinates in the normal and tangent directions, respectively, and $r$ is a scaling factor such that $r \rightarrow 0$. Let us choose the normal vector $\vec{n}$ so that it points in the direction of the disordered region $D$, then the point $\vec{H}(r, s, t)$ belongs to $D$ if $s \geq 0$.

Theorem 5.1. Let $\vec{H}(r, s, t)$ be defined as above. The asymptotics of the free energy of the 6-vertex model in the limit $r \rightarrow 0$ is given by

$$
f(\vec{H}(r, s, t))=f_{\text {lin }}(\vec{H}(r, s, t))+\eta(s, t) r^{3}+O\left(r^{5}\right),
$$

where $f_{\text {lin }}(H, V)=-\ln (a)-H-V$ and

$$
\eta(s, t)=-\kappa\left(\theta s+t^{2}\right)^{3 / 2} .
$$

Here the constants $\kappa$ and $\theta$ depend on the Boltzmann weights of the model and on $\left(H_{0}, V_{0}\right)$ and are given by

$$
\kappa=\frac{16}{3 \pi} \partial_{H}^{2} g\left(H_{0}, V_{0}\right)
$$

and

$$
\theta=\frac{4+\left(\partial_{H} g\left(H_{0}, V_{0}\right)\right)^{2}}{2 \partial_{H}^{2} g\left(H_{0}, V_{0}\right)}
$$

where $g(H, V)$ is defined in (5.1).

Moreover, $\partial_{H}^{2} g\left(H_{0}, V_{0}\right)>0$ and, therefore, $\theta>0$.

We refer the reader to $[\mathrm{P}]$ for the details. 


\subsection{The scaling in the tentacle}

Assume that $a>b$. The theorem below describes the asymptotic of the free energy function when $H \rightarrow+\infty$ and

$$
\frac{1}{2} \ln (b / a)-\frac{c^{2}}{2 a b} e^{-2 H} \leq V \leq \frac{1}{2} \ln (b / a)+\frac{c^{2}}{2 a b} e^{-2 H}, \quad H \longrightarrow \infty .
$$

These values of $(H, V)$ describe points inside the right "tentacle" on the Fig. 5 .

Let us parameterize these values of $V$ as

$$
V=\frac{1}{2} \ln (b / a)+\beta \frac{c^{2}}{2 a b} e^{-2 H},
$$

where $\beta \in[-1,1]$.

Theorem 5.2. When $H \rightarrow \infty$ and $\beta \in[-1,1]$ the asymptotic of the free energy is given by the following formula:

$$
\begin{aligned}
f(H, V)=- & \frac{1}{2} \ln (a b)-H- \\
& \frac{c^{2}}{2 a b} e^{-2 H}\left(\beta+\frac{2}{\pi} \sqrt{1-\beta^{2}}-\frac{2}{\pi} \beta \arccos (\beta)\right)+O\left(e^{-4 H}\right),
\end{aligned}
$$

Proof. From the integral equation for $\rho(z)$ we can derive the large $H$ asymptotics of the density function:

$$
\rho(z)=\frac{1}{z}+\frac{2 \Delta \alpha}{z^{2}}+\ldots, \quad|z| \rightarrow \infty
$$

The integration contour is symmetric with respect to complex conjugation $z \rightarrow \bar{z}$. The contour is a small deformation of the segment of the circle of radius $e^{2 H}$ centered at the origin with endpoints having arguments $\pm \pi \alpha$.

For large $H$ the free energy function is given by

$$
\begin{aligned}
f(H, V)= & \min _{0 \leq \alpha \leq 1}(-\ln a-H-(1-2 \alpha) V \\
& \left.-\frac{1}{2 \pi i} \int_{C} \ln \left(\frac{b}{a}+\frac{c^{2}}{a^{2} z-a b}\right) \rho(z) d z\right) .
\end{aligned}
$$

As $H \rightarrow \infty$ the density is given by (5.5) and, taking into account the asymptotical description of the contour of integration we obtain

$$
\begin{aligned}
f(H, V)= & \min _{\alpha}(-\ln a-H-V+\alpha(2 V-\ln (b / a)) \\
& \left.-\frac{c^{2}}{a b} e^{-2 H} \kappa(\alpha)+O\left(e^{-4 H}\right)\right),
\end{aligned}
$$

where

$$
e^{-2 H} \kappa(\alpha)=\int_{C} \frac{d w}{w^{2}}
$$


This integral is easy to compute:

The minimum occur at

$$
\kappa(\alpha)=\frac{\sin (\pi \alpha)}{\pi}
$$

$$
\cos (\pi \alpha)=\frac{a b}{c^{2}}(2 V-\ln (b / a)) e^{2 H}
$$

or, at

$$
\cos (\pi \alpha)=\beta
$$

The formula (5.5) follows after the substitution of this into the expression for $f(H, V)$.

\subsection{The 5-vertex limit}

The 5-vertex model can be obtained as the limit of the 6-vertex model when $\Delta \rightarrow \infty$. Magnetic fields in this limit behave as follows:

- $a>b+c$. In the parameterization (4.2) after changing variables $H=\frac{\eta}{2}+l$, and $V=-\frac{\eta}{2}+m$ take the limit $\eta \rightarrow \infty$ keeping $\lambda$ fixed. The weights will converge (up to a common factor) to:

$$
a_{1}: a_{2}: b_{1}: b_{2}: c_{1}: c_{2} \rightarrow e^{\lambda+l+m}: e^{\lambda-l-m}:\left(e^{\lambda}-e^{-\lambda}\right) e^{l-m}: 0: 1: 1
$$

- $a+c<b$. In the parameterization (4.3) after changing variables $H=\frac{\eta}{2}+l$, and $V=\frac{\eta}{2}+m$ take the limit $\eta \rightarrow \infty$ keeping $\xi=\lambda-\eta$ fixed. The weights will converge (up to a common factor) to:

$$
a_{1}: a_{2}: b_{1}: b_{2}: c_{1}: c_{2} \rightarrow\left(e^{\xi}-e^{-\xi}\right) e^{l+m}: 0: e^{\xi+l-m}: e^{\xi-l+m}: 1: 1
$$

The two limits are related by inverting horizontal arrows. From now on we will focus on the 5 -vertex model obtained by the limit from the 6-vertex one when $a>b+c$.

The phase diagram of the 5-vertex model is easier then the one for the 6-vertex model but still sufficiently interesting. Perhaps the most interesting feature is that the existence of the tricritical point in the phase diagram.

We will use the parameter

$$
\gamma=e^{-2 \lambda}
$$

Notice that $\gamma<1$.

The frozen regions on the phase diagram of the 5-vertex model, denoted on Fig. 11 as $A_{1}, A_{2}$, and $B_{1}$, can be described by the following inequalities:

$$
\begin{array}{ll}
A_{1} \text {-region: } & m \geq-l, \quad l \leq 0, \\
& e^{2 m} \geq 1-\gamma\left(1-e^{-2 l}\right), \quad l>1 ; \\
A_{2} \text {-region: } & m \leq-l, \quad l \leq 0, \\
& e^{2 m} \leq 1-\frac{1}{\gamma}\left(1-e^{-2 l}\right), \quad l>1 ; \\
B_{1} \text {-region: } & \left(e^{2 l}-\frac{1}{1-\gamma}\right)\left(e^{-2 m}-\frac{1}{1-\gamma}\right) \geq \frac{\gamma}{(1-\gamma)^{2}}, \quad e^{2 l}>\frac{1}{1-\gamma} ;
\end{array}
$$




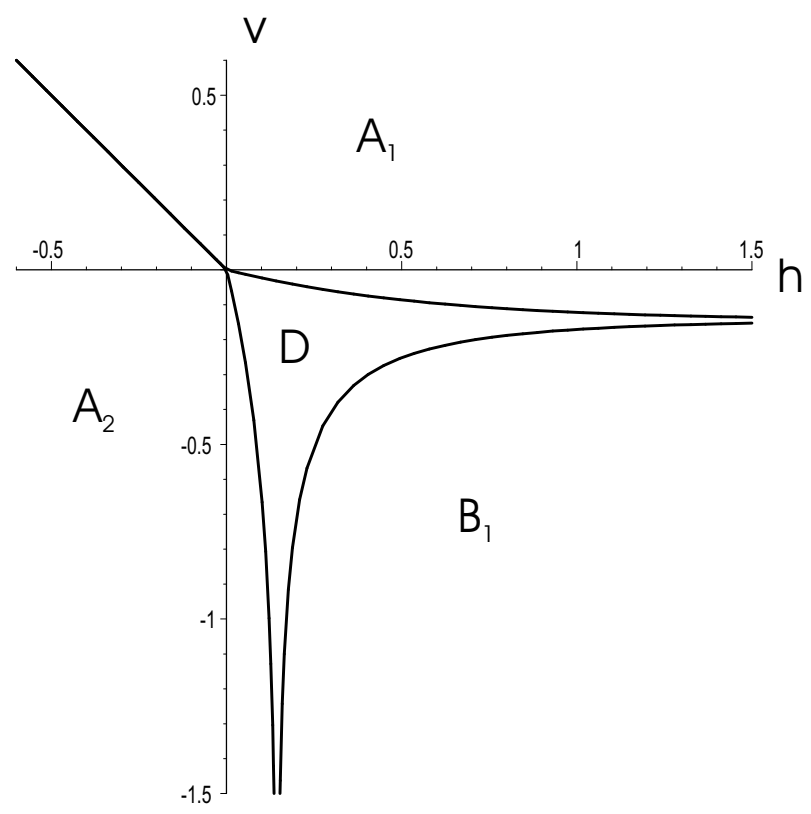

Figure 11: The phase diagram of the 5-vertex model with $\gamma=1 / 4\left(\beta=e^{-2 h}\right)$.

\subsection{The asymptotic of the free energy near the tricritical point in the 5-vertex model}

The disordered region $D$ near the tricritical point forms a corner

$$
-\frac{1}{\gamma} l+O\left(l^{2}\right) \leq m \leq-\gamma l+O\left(l^{2}\right), \quad h \rightarrow 0+.
$$

The angle $\theta$ between the boundaries of the disordered region at this point is given by

$$
\cos (\theta)=\frac{2 \gamma}{1+\gamma^{2}}
$$

One can argue that the finiteness of the angle $\theta$ manifests the presence of interaction in the model. In comparison, translationary invariant dimer models can only have cusps as such singularities.

As it follows from results [HWKK] the limit from the 6-vertex model to the 5-vertex model commutes with the thermodynamical limit and for the free energy of the 5-vertex model we can use the formula

$$
f(l, m)=\lim _{\eta \rightarrow+\infty}(F(\eta / 2+l,-\eta / 2+m)-F(\eta / 2,-\eta / 2)),
$$

where $F(H, V)$ is the free energy of the 6-vertex model.

Theorem 5.3. Let $\gamma \leq k \leq \frac{1}{\gamma}$ and

$$
m=-k l,
$$


The asymptotics of the free energy along this ray inside the corner near the tricritical point is given by

$$
f(l,-k l)=c_{1}(k, \gamma) l+c_{2}(k, \gamma) l^{5 / 3}+O\left(l^{7 / 3}\right) .
$$

where

$$
c_{1}(k, \gamma)=\frac{1}{1-\gamma}(-(1+k)(1+\gamma)+4 \sqrt{k \gamma})
$$

and

$$
c_{2}(k, \gamma)=(6 \pi)^{2 / 3} \frac{2 \gamma^{5 / 6}(1-\gamma) k^{3 / 2}(\sqrt{k}-1 / \sqrt{\gamma})^{4 / 3}}{5(\sqrt{k}-\sqrt{\gamma})^{4 / 3}} .
$$

The proof is computational. The details can be found in $[\mathrm{P}]$.

The scaling along any ray inside the corner near the tricritical point in the 6-vertex model differ from this only by in coefficients. The exponent $h^{5 / 3}$ is the same. The details will be given in a separate publication.

\subsection{The limit $\Delta \rightarrow-1^{-}$}

If $m=1$, the region $A$ consists of one point located at the origin. When $\eta \rightarrow 0+$ we have $\Delta \rightarrow-1-$ and $m \rightarrow 1-$. Moreover, $K^{\prime} \rightarrow \pi / 2, K \rightarrow \frac{\pi^{2}}{2 \eta}$, and $\frac{1}{\operatorname{dn}(u \mid 1-m)} \sim 1+\frac{1}{2}(1-m) \sin ^{2}(u)$. Since $\cosh ^{-1}(x) \sim$ $\pm \sqrt{2(x-1)}$, when $x \rightarrow 1+$, we have

$$
\theta_{0}=\frac{|b-a| \eta}{a+b}
$$

Since $\Xi(\varphi)$ is an odd function we obtain the following asymptotic of $\Xi[\mathrm{LW}]$ :

$$
\Xi(\varphi) \sim 4 e^{-\frac{\pi^{2}}{2 \eta}} \sin \left(\frac{\pi}{2 \eta} \varphi\right) .
$$

In this limit the antiferromagnetic region degenerates into the origin $H=0$ and $V=0$ exponentially fast. We note that the point $H=V=0$ is special for $|\Delta|<1$.

\subsection{The convexity}

The following identity holds in the region $D[\mathrm{NK}]$ :

$$
f_{H, H} f_{V, V}-f_{H, V}^{2}=\left(\frac{2}{\pi g}\right)^{2} .
$$

Here $g=\frac{1}{2 D_{0}^{2}}$. The constant $D_{0}$ does not vanish in the $D$-region including its boundary. It is determined by the solution to the integral equation for the density $\rho(z)$ (see Appendix C).

Directly from the definition of the free energy we have

$$
f_{H, H}=\lim _{N, M \rightarrow \infty} \frac{<(n(L)-n(R))^{2}>}{N M},
$$

where $n(l)$ and $n(r)$ are the number of arrows pointing to the left and the number of arrows pointing to the right, respectively. 
Therefore, the matrix $\partial_{i} \partial_{j} f$ of second derivatives with respect to $H$ and $V$ is positive definite.

As it follows from the asymptotical behavior of the free energy near the boundary of the $D$-phase, despite the fact that the Hessian is nonzero and finite at the boundary of the interface, the second derivative of the free energy in the transversal direction at a generic point of the interface develops a singularity.

\section{The Legendre Transform of the Free Energy}

The Legendre transform of the free energy

$$
\sup _{H, V}(x H+y V+f(H, V))
$$

as a function of $(x, y)$ is defined for $-1 \leq x, y \leq 1$.

The variables $x$ and $y$ are known as polarizations and are related to the slope of the Gibbs measure as $x=2 h-1$ and $y=2 v-1$. We will write the Legendre transform of the free energy as a function of $(h, v)$

$$
\sigma(h, v)=\sup _{H, V}((2 h-1) H+(2 v-1) V+f(H, V)) .
$$

$\sigma(h, v)$ is defined on $0 \leq h, v \leq 1$.

For the periodic boundary conditions the surface tension function has the following symmetries:

$$
\sigma(x, y)=\sigma(y, x)=\sigma(-x,-y)=\sigma(-y,-x) .
$$

The last two equalities follow from the fact that if all arrows are reversed, $\sigma$ is the same, but the signs of $x$ and $y$ are changed. It follows that $\sigma_{h}(h, v)=\sigma_{v}(v, h)$ and $\sigma_{v}(h, v)=\sigma_{h}(v, h)$.

The function $f(H, V)$ is linear in the domains that correspond to conic and corner singularities of $\sigma$. Outside of these domains (in the disordered domain $D$ ) we have

$$
\nabla \sigma \circ \nabla f=\operatorname{id}_{D}, \nabla f \circ \nabla \sigma=\operatorname{id}_{\nabla f(D)} .
$$

Here we gradient of a function as a mapping $\mathbb{R}^{2} \rightarrow \mathbb{R}^{2}$.

When the 6-vertex model is formulated in terms of the height function, the Legendre transform of the free energy can be regarded as a surface tension. The surface in this terminology is the graph of of the height function.

\section{1}

Now let us describe some analytical properties of the function $\sigma(h, v)$ is obtained as the Legendre transform of the free energy. The Legendre transform maps the regions where the free energy is linear with the slope $( \pm 1, \pm 1)$ to the corners of the unit square $\mathscr{D}=\{(h, v) \mid \quad 0 \leq h \leq 1,0 \leq v \leq 1\}$. For example, the region $A_{1}$ is mapped to the corner $h=1$ and $v=1$ and the region $B_{1}$ is mapped to the corner $h=1$ and $v=0$. The Legendre transform maps the tentacles of the disordered region to the regions adjacent to the 
boundary of the unit square. For example, the tentacle between $A_{1}$ and $B_{1}$ frozen regions is mapped into a neighborhood of $h=1$ boundary of $\mathscr{D}$, i.e. $h \rightarrow 1$ and $0<v<1$.

Applying the Legendre transform to asymptotics of the free energy in the tentacle between $A_{1}$ and $B_{1}$ frozen regions we get

$$
H(h, v)=-\frac{1}{2} \ln \left(\frac{\pi a b}{c^{2}} \frac{1-h}{\sin \pi(1-v)}\right), \quad V(h, v)=\frac{1}{2} \ln (b / a)+\frac{\pi}{2}(1-h) \cot (\pi(1-v)),
$$

and

$$
\sigma(h, v)=(1-h) \ln \left(\frac{\pi a b}{c^{2}} \frac{1-h}{\sin (\pi(1-v))}\right)-(1-h)+v \ln (b / a)-\ln (b),
$$

Here $h \rightarrow 1-$ and $0<v<1$. From (6.3) we see that $\sigma(1, v)=v \ln (b / a)-\ln (b)$, i.e. $\sigma$ is linear on the boundary $h=1$ of $\mathscr{D}$. Therefore, its asymptotics near the boundary $h=1$ is given by

$$
\sigma(h, v)=v \ln (b / a)-\ln (b)+(1-h) \ln (1-h)+O(1-h),
$$

as $h \rightarrow 1-$ and $0<v<1$. We note that this expansion is valid when $(1-h) / \sin (\pi(1-v)) \ll 1$.

Similarly, considering other tentacles of the region $D$, we conclude that the surface tension function is linear on the boundary of $\mathscr{D}$.

\section{2}

Next let us find the asymptotics of $\sigma$ at the corners of $\mathscr{D}$ in the case when all points of the interfaces between frozen and disordered regions are regular, i.e. when $\Delta<1$. We use the asymptotics of the free energy near the interface between $A_{1}$ and $D$ regions (5.2).

First let us fix the point $\left(H_{0}, V_{0}\right)$ on the interface and the scaling factor $r$ in (5.2). Then from the Legendre transform we get

$$
1-h=-\frac{3}{4} r \frac{\kappa\left(\theta s+t^{2}\right)^{1 / 2}}{\left(\partial_{H} g\right)^{2}+4}\left(\theta \partial_{H} g+4 r t\right)
$$

and

$$
1-v=-\frac{3}{2} r \frac{\kappa\left(\theta s+t^{2}\right)^{1 / 2}}{\left(\partial_{H} g\right)^{2}+4}\left(-\theta+r \partial_{H} g t\right)
$$

It follows that

$$
\frac{1-h}{1-v}=\frac{\theta \partial_{H} g+4 r t}{2\left(-\theta+r t \partial_{H} g\right)}
$$

In the vicinity of the boundary $r \rightarrow 0$ and, hence,

$$
\frac{1-h}{1-v}=-\frac{\partial_{H} g}{2}=\frac{1-b / a e^{-2 V_{0}}}{1-b / a e^{-2 H_{0}}}
$$

as $h, v \rightarrow 1$. Thus, under the Legendre transform, the slope of the line which approaches the corner $h=v=1$ depends on the boundary point on the interface between the frozen and disordered regions. 
It follows that the first terms of the asymptotics of $\sigma$ at the corner $h=v=1$ are given by

$$
\sigma(h, v)=-\ln a-2(1-h) H_{0}(h, v)-2(1-v) V_{0}(h, v),
$$

where $H_{0}(h, v)$ and $V_{0}(h, v)$ can be found from (6.4) and $g\left(H_{0}, V_{0}\right)=0$.

When $|\Delta| \leq 1$ the function $\sigma$ is strictly convex and smooth for all $0<h, v<1$. It develops conical singularities near the boundary.

When $\Delta<-1$, in addition to the singularities on the boundary, $\sigma$ has a conical singularity at the point $(1 / 2,1 / 2)$. It corresponds to the "central flat part" of the free energy $f$, see Fig. 8 .

When $\Delta>1$ the function $\sigma$ has corner singularities along the boundary as in the other cases. In addition to this, it has a corner singularity along the diagonal $v=h$ if $a>b$ and $v=1-h$ if $a<b$. We refer the reader to $[\overline{\mathrm{BS}}]$ for further details on singularities of $\sigma$ in the case when $\Delta>1$.

\section{The Thermodynamic Limit and the Variational Principle for Fixed Boundary Conditions}

\subsection{The Variational Principle}

\subsection{1}

Let $\sigma$ be the surface tension function of the 6-vertex model with the periodic boundary conditions defined in (6.1). We consider the functional

$$
I[\varphi]=\int_{D} \sigma(\nabla \varphi) d^{2} x+\lambda \int_{D} \varphi d^{2} x
$$

Let $h(x, y)$ be a minimizer of this functional on the space $L\left(D, \varphi_{0}\right)$ of functions nondecreasing in $x$ and $y$ directions and satisfying the condition

$$
\varphi(x, y)-\varphi\left(x^{\prime}, y^{\prime}\right) \leq x-x^{\prime}+y-y^{\prime}
$$

and the boundary condition

$$
\left.\varphi\right|_{\partial D}=\varphi_{0} .
$$

Notice that height functions are Lipschitz with $\left|\varphi(x, y)-\varphi\left(x^{\prime}, y^{\prime}\right)\right| \leq 2 \max \left(\left|x-x^{\prime}\right|,\left|y-y^{\prime}\right|\right)$.

Proposition 7.1. The functional I $[\varphi]$ has unique minimizer.

Indeed, since $\sigma$ is convex, the minimizer is unique when it exists. The existence of the minimizer follows from compactness of the space $L\left(D, \varphi_{0}\right)$ in the sup norm. The arguments are completely parallel to those in $[\mathrm{CKP}]$. 


\subsection{2}

If the vector $\nabla h(x, y)$ is not a singular point of $\sigma$, the minimizer $h$ satisfies the Euler-Lagrange equation in a neighborhood of $(x, y)$

$$
\operatorname{div}(\nabla \sigma \circ \nabla h)=\lambda
$$

We can also rewrite this equation in the form

$$
\nabla \sigma(\nabla h(x, y))=\frac{\lambda}{2}(x, y)+\left(-g_{y}(x, y), g_{x}(x, y)\right),
$$

where $g$ is an unknown function such that $g_{x y}(x, y)=g_{y x}(x, y)$. It is determined by the boundary conditions for $h$.

Applying (6.2), we see that

$$
\nabla h(x, y)=\nabla f\left(\frac{\lambda}{2} x-g_{y}(x, y), \frac{\lambda}{2} y+g_{x}(x, y)\right) .
$$

From the definition of the slope, see (4.1) we have $0 \leq f_{H} \leq 1$ and $0 \leq f_{V} \leq 1$. Thus, if the minimizer $h$ is differentiable at $(x, y)$, it satisfies the constrains $0 \leq h_{x} \leq 1$ and $0 \leq h_{y} \leq 1$.

\subsection{Large deviations}

The following statement is a minor variation of the theorem 4.3 from [CKP].

Theorem 7.2. Let $N \rightarrow \infty, \lambda$ be finite and $q=\exp \left(\frac{\lambda}{N}\right)$ then the sequence of random normalized height functions $h_{N}$ converges in probability to the minimizer of (7.1). The rate of convergence is exponential of $N^{2}$.

The minimizer of the variational problem (7.1) is called the limit shape of the height function.

This theorem is the manifestation of the general philosophy of the large deviations principle. The probability of a having state with the height function $h$ has the has the following asymptotic $N \rightarrow \infty$ :

$$
\operatorname{Prob}(h) \sim \exp \left(\lambda N^{2} \int_{D} h d^{2} x+N^{2} \int_{D} \sigma(\nabla h) d^{2} x\right) .
$$

Here $\sigma$ is the surface tension function for the periodic boundary conditions. Clearly this probability has maximum at the limit shape. States with the height function, which macroscopically differ from the limit shape, should be expected to be exponentially improbable. The theorem states that this is exactly what is taking place.

\section{The limit $|\lambda| \rightarrow \infty$}

\subsection{Minimal and maximal height functions}

The space of normalized height functions on $L_{N, M}$ has the partial order described in section 2. Denote the minimal and maximal normalized height functiions with respect to this partial order $h^{\min }$ and $h^{\max }$, respectively. 
For two functions $h_{1}, h_{2}$ on $D$ define the distance

$$
\operatorname{dist}\left(h_{1}, h_{2}\right)=\sup _{x \in D}\left|h_{1}(x)-h_{2}(x)\right|
$$

Similarly define the the distance between two functions on the boundary of $D$.

Let $h_{0}^{(N)}$ be a sequence of functions on the boundary of $D$ converging to $\varphi_{0}$, and such that $h_{0}^{(N)}$ is a boundary of a normalized height function of $L_{N, M}$. Denote $h_{\min }^{(N)}$ and $h_{m a x}^{(N)}$ the minimum and maximum height functions from $L_{N, M}\left(h_{0}^{(N)}\right)$. The following is clear:

Proposition 8.1. Let $\varphi_{\min }$ and $\varphi_{\max }$ be minimal and maximal height functions with the boundary condition $\varphi_{0}$. Then, $\varphi_{\min }=\lim _{N \rightarrow \infty} h_{N}^{\min }$ and $\varphi_{\max }=\lim _{N \rightarrow \infty} h_{N}^{\max }$ with respect to the distance (8.1).

The functions $\varphi_{\min }$ and $\varphi_{\max }$ minimize the functionals

$$
\pm \int_{D} \varphi d^{2} x
$$

in the sapec $H\left(\varphi_{0}\right)$.

Let us assume that the boundary conditions are critical, that is $\varphi_{0}$ is piece-wise linear, non-decreasing in $x$ and $y$ direction with the slope 0 or 1 . In this case the minimum and maximum height functions are piecewise linear functions, such that each linear part has the slope either 0 or \pm 1 along coordinate axes.

We will say a point $(x, y)$ is regular if at this point the function $\varphi_{\min }$ is differentiable. For critical boundary conditions regular points form regions with piece-wise linear boundary where the function $\varphi_{\min }$ has a constant slope. We will call them linear domains.

Points where the gradient of $\varphi_{\min }$ is discontinuous will be called singular points. For critical boundary conditions singular points are the points where several linear domains meet. The valency of a singular point is the number of linear domains which meet at this point. For generic critical boundary conditions the valency of each critical point is at most three.

The list of all possible phases at a tricritical point of the 6-vertex model with generic critical boundary conditions is given on Fig. 12 .

\subsection{The asymptotic of the minimizer when $|\lambda| \rightarrow \infty$}

Here we study the asymptotic of minimizer of $I_{\lambda}[\varphi]$ as $\lambda \rightarrow \pm \infty$. It is more convenient to divide $I_{\lambda}$ by $|\lambda|$, so we are looking for the asymptotics of the minimizer $h_{\lambda}$ of

$$
I_{\lambda}^{ \pm}[\varphi]=\frac{1}{|\lambda|} \int_{D} \sigma(\nabla \varphi) d^{2} x \pm \int_{D} \varphi d^{2} x .
$$

Let us focus on the limit $\lambda \rightarrow+\infty$. The limit $\lambda \rightarrow-\infty$ can be treated similarly.

When $\lambda \rightarrow+\infty$, the minimizer $h_{\lambda}$ approaches the minimal height function $\varphi_{\min }$ described above. Let us look for the asymptotical formula for the minimizer in a small neighborhood of a point $\left(x_{0}, y_{0}\right)$ of the form

$$
h_{\lambda}(x, y)=\varphi_{\min }(x, y)+\frac{1}{\lambda} H_{\lambda}\left(\lambda\left(x-x_{0}\right), \lambda\left(y-y_{0}\right)\right),
$$



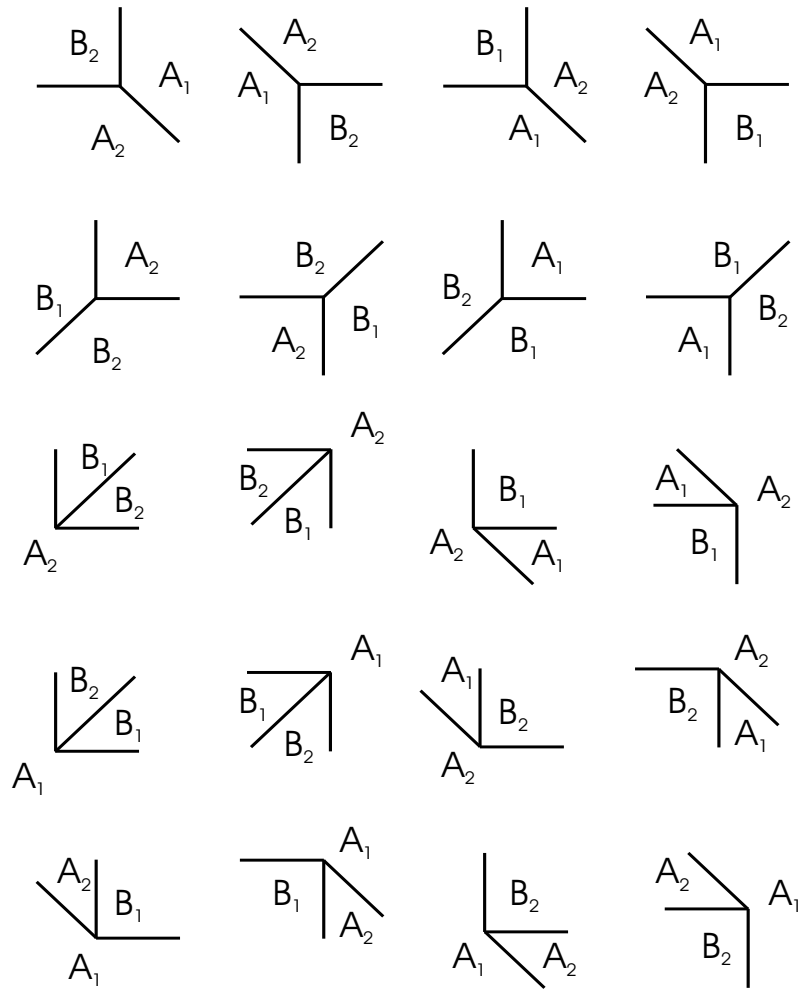

Figure 12: Possible phases at a tricritical point of the 6-vertex model.

where $H_{\lambda}=H+o(1)$ as $\lambda \rightarrow+\infty$.

Let $z=\left(z_{1}, z_{2}\right)=(r, s)=\left(\lambda\left(x-x_{0}\right), \lambda\left(y-y_{0}\right)\right)$ and $x=\left(x_{1}, x_{2}\right)=(x, y)$. Because $\varphi_{\min }$ is linear, its second derivatives vanish and we can rewrite the Euler-Lagrange equation (7.2) as

$$
\frac{\partial^{2} H_{\lambda}}{\partial z_{i} \partial z_{j}} \frac{\partial^{2} \sigma}{\partial u_{i} \partial u_{j}}\left(\nabla_{x} \varphi_{\min }(x, y)+\nabla_{z} H_{\lambda}(z)\right)=1 .
$$

Notice that for critical boundary conditions the function $\nabla \varphi_{\min }$ is piece-wise constant.

Now assume that $\left(x_{0}, y_{0}\right)$ is a singular point, i.e. a point where two or more linear domains of $\varphi_{\min }$ meet. Recall that for generic critical boundary conditions only two or three linear domains can meet at a point. Taking the limit $\lambda \rightarrow \infty$ in (8.2) we obtain.

Proposition 8.2. Let $h_{\lambda}$ be the minimizer of $I_{\lambda}^{+}$and $\left(x_{0}, y_{0}\right)$ be a singular point, then for each $(s, t) \in \mathbb{R}^{2}$ there exits

$$
H(r, s)=\lim _{\lambda \rightarrow \infty} \lambda\left(h_{\lambda}\left(x_{0}+\frac{r}{\lambda}, y_{0}+\frac{s}{\lambda}\right)-\varphi^{\min }\left(x_{0}, y_{0}\right)\right)
$$

This function is the solution to

$$
\operatorname{div}(\nabla \sigma \circ(\nabla H))=1
$$


with the boundary conditions

$$
H(r, s) \rightarrow k_{i} r+l_{i} s
$$

as $(r, s) \rightarrow \infty$ along a ray in the $i$-th linear domain adjacent to $\left(x_{0}, y_{0}\right)$. Here $\left(k_{i}, l_{i}\right)=\nabla \varphi_{\text {min }}$ in the $i$-th linear domain and since the boundary conditions are critical, $k_{i}, l_{i}=0,1$.

Conjecture 8.1. The function $H$ is once differentiable. There is a smooth curve separating $\mathbb{R}^{2}$ into regions where $H$ is linear and regions where $H$ is smooth with positive definite matrix of second derivatives.

Remark 2. For non-generic boundary conditions in the thermodynamical limit more then three linear domains can met at one point. In this case one should expect that the conjecture still holds with more then three linear domains meeting at a singular point.

\subsection{The asymptotic near double degenerate singular points}

\subsection{1}

A height function defines the surface $z=h(x, y)$ in $\mathbb{R}^{3}$. Regions where $h$ is linear are planes. Here we will describe the solution to the equation $(8.4)$ with the boundary conditions $(8.5)$ in the case when there are only two asymptotic planes meeting at $\left(x_{0}, y_{0}\right)$.

Let $\left(k_{1}, k_{2}\right)$ and $\left(l_{1}, l_{2}\right)$ be the directions of the steepest assent of these planes. The numbers $k_{i}$ and $l_{i}$ are either 0 or 1 since we assume critical boundary conditions and therefore $\varphi_{\min }$ is piece-wise linear with slope $(0,0),(0,1),(1,0)$ or $(1,1)$.

The function $H(r, s)$, defined in $(8.3)$, has the asymptotic conditions $(8.5)$ determined by these planes. The function $H(r, s)$ is also invariant with respect to translations in $m=(k-l)^{\perp}$-direction.

Thus, we are looking for a function $\kappa$ such that

$$
H(r, s)=\kappa\left(\left(k_{1}-l_{1}\right) r+\left(k_{2}-l_{2}\right) s\right),
$$

which satisfies the differential equation (8.4) with the asymptotic conditions (8.5).

Let us introduce

$$
S(t)=\left.\sum_{i, j=1,2}(k-l)_{i}(k-l)_{j} \frac{\partial^{2} \sigma}{\partial u_{i} \partial u_{j}}(u)\right|_{u=(k-l) t} .
$$

Then the differential equation for $H$ becomes the first order ODE for the function $\kappa^{\prime}$

$$
\kappa^{\prime \prime}(t) S\left(\kappa^{\prime}(t)\right)=1
$$

Integrating it, we obtain the equation defining the function $g(t)=\kappa^{\prime}(t)$ implicitly

$$
\sum_{i=1,2}\left(k_{i}-l_{i}\right) \frac{\partial \sigma}{\partial u_{i}}(g(t)(k-l))=t+C
$$

with some constant $C$. 


\subsection{2}

For the domain wall boundary conditions minimal and maximal height functions are shown on Fig. 13 . In this case the boundary between two linear domains is a line in the direction $(1,-1)$ for the minimal height function and is the line in the direction $(1,1)$ for the maximal height function.

Thus, for these boundary conditions the function $H$ describing the asymptotic of the minimizer is constant in the direction $(1,-1)$ when $\lambda \rightarrow+\infty$ and it is constant in the $(1,1)$-direction if $\lambda \rightarrow-\infty$.

Let $H(r, s)=\kappa(s+r)$ be the solution to (8.6) which is invariant with respect to translations in the $(1,-1)$ direction. The symmetries of the Legendre transform $\sigma$ of the free energy imply that $\sigma_{h}(h, v)=\sigma_{v}(v, h)$. Using the equation (8.7) and this symmetry of $\sigma$, we obtain

$$
\frac{\partial \sigma}{\partial u_{1}}(g(t), g(t))=t / 2+C / 2 \text {. }
$$

Taking into account $(\underline{6.2)}$ we obtain

$$
g(t)=\partial_{H} f(t / 2+C / 2, t / 2+C / 2)=\partial_{V} f(t / 2+C / 2, t / 2+C / 2)
$$

and, hence,

$$
\kappa(t)=f(t / 2+C / 2, t / 2+C / 2) .
$$

In the case of two asymptotic planes we have an additional symmetry of $H(r, s)$ with respect to the intersection line of these planes, i.e. $\kappa(t)=\kappa(-t)$. The free energy $f$ also has the symmetry $f(x, y)=$ $f(-y,-x)$. Therefore $C=0$ and we proved the following statement.

Theorem 8.3. For $D W$ boundary conditions the minimizer $h_{\lambda}$ when $\lambda \rightarrow+\infty$ has the following asymptotic when $x=x_{0}+r / \lambda, y=-x_{0}+s / \lambda$ :

$$
h_{\lambda}(x, y)=\varphi_{\min }+\frac{1}{\lambda} f\left(\frac{r+s}{2}, \frac{r+s}{2}\right)+o\left(\frac{1}{\lambda^{2}}\right) .
$$

If $x=x_{0}+r / \lambda$ and $y=x_{0}+s / \lambda$ and $\lambda \rightarrow-\infty$ the asymptotic of the minimizer $h_{\lambda}$ is given by

$$
h_{\lambda}(x, y)=\varphi_{\min }+\frac{1}{\lambda} f\left(\frac{r-s}{2},-\frac{r-s}{2}\right)+o\left(\frac{1}{\lambda^{2}}\right) .
$$

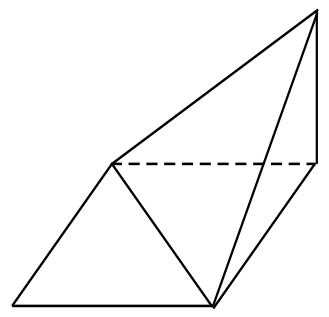

$\mathrm{h}_{\min }$

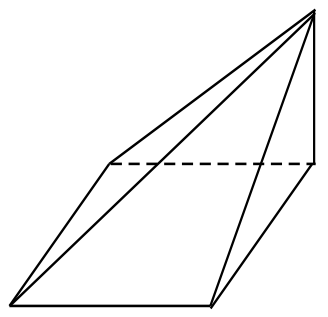

$\mathrm{h}_{\max }$

Figure 13: The minimum and maximum height functions for the DW boundary conditions. 


\subsection{3}

When $c>a+b$ (which is equivalent to $\Delta<0$ ) the height function $h_{\lambda}$ develops extra linear domains known as facets. These are the regions of the antiferroelectric phase discussed in section 4.3.2. In dimer models this phenomenon is studied in [KOS].

The facets also appear in the function $H$ describing the asymptotic of the minimizer as $\lambda \rightarrow \infty$.

For the function $H$ describing the asymptotic near a double singular point $\left(x_{0}, y_{0}\right)$ where two linear domains meet along the diagonal $x+y=0$ the facet is a strip

$$
|s+t| \leq R,
$$

in coordinates $x=x_{0}+\frac{s}{\lambda}, y=-x_{0}+\frac{t}{\lambda}$. Its width $R$ is given by the formula

$$
R=\sqrt{2}\left|\Xi\left(\frac{\eta+\theta_{0}}{2}\right)\right| \text {. }
$$

In the limit $\Delta \rightarrow-1-$ or $\eta \rightarrow 0+$ the asymptotic of $\Xi$ gives the following asymptotical value of $R$ :

$$
R=4 \sqrt{2} e^{-\frac{\pi^{2}}{2 \eta}} \sin \left(\frac{\pi \max (a, b)}{2(a+b)}\right)(1+O(\eta)) .
$$

The free energy $f$ is linear in the antiferromagnetic region. It is growing with exponent $3 / 2$ in the normal direction to the boundary outside of the antiferromagnetic region. This agrees with the PokrovskyTalapov law which states that $f$ should be growing with exponent $3 / 2$ in the normal direction to the boundary of the facet $[\mathrm{PT}]$. In particular, $h$ grows with the exponent $3 / 2$ in the $(1,1)$-direction near the boundary of the $c$-droplet far enough from the boundary of the square. For $s \rightarrow R+0$ we have

$$
h_{\lambda}\left(x_{0}+\frac{s}{\lambda},-x_{0}\right)=h_{0}+\frac{\kappa}{\lambda}(s-R)^{3 / 2},
$$

where $h_{0}$ is the value of the height function at the boundary of the facet and $\kappa$ is a constant which can be computed explicitly.

\section{Conclusion}

\subsection{Correlation functions in the bulk}

As we have seen in the previous section at the macroscopical distances in the thermodynamical limit the height function is deterministic and is the minimizer for the variational problem (7.1).

But the height function at smaller distances remain random. Their fluctuations are described by the asymptotical behavior of correlation functions in the thermodynamical limit. These asymtotics have been studied extensively in dimer models which describe the $\Delta=0$ case of the 6-vertex model.

The thermodynamical limit of correlations functions in the bulk describes translationary invariant Gibbs measure with given polarization. These asymptotics of correlation functions have been studied a lot using various methods which are essentially based on representation theory of affine quantum groups. 
The exact computation of the asymptotic of correlation functions for local observable separated by large distances on the lattice remain one of the main problems. On the other hand this is one of the most important physically relevant information about correlation functions. Some information about this asymptotic of correlation functions can be obtained using the arguments of finite-size scaling and the assumption of conformal invariance of the leading terms of the asymptotic.

Let us consider the 6-vertex configurations of paths which may end at some edges. The weight of such configurations is given the same product of weights as before. Define local observables $\tau_{e}$ and $\tau_{e}^{*}$ as

$$
\begin{gathered}
\tau_{e}(S)= \begin{cases}1, & \text { if a path going up starts at } e \\
0, & \text { otherwise }\end{cases} \\
\tau_{e}^{*}(S)= \begin{cases}1, & \text { if a path going down starts at } e \\
0, & \text { otherwise }\end{cases}
\end{gathered}
$$

The value of a product of such observable when each of the factors correspond to a different edge is the product of values of observables.

The following formulae were obtained in $[\overline{B I R}]$ for $H=0$ and when all edges are vertical on the same row using the finite-size scaling and the assumption of conformal invariance:

$$
\begin{aligned}
<\sigma_{e_{1}} \sigma_{e_{2}}>\simeq & <\sigma_{e}>^{2}+\frac{A}{d^{2}}+\frac{B \cos \left(2 k_{F} d\right)}{d^{\alpha}}+\ldots \\
& <\tau_{e_{1}}^{*} \tau_{e_{2}}>\simeq \frac{C}{d^{\frac{1}{\alpha}}}+\ldots
\end{aligned}
$$

Here $j$ is the distance between $e_{1}$ and $e_{2}, \alpha=2 \pi^{2} \rho(\xi)^{2}$. The terms denoted by $\ldots$ are with higher powers of $d^{-2}$ and $d^{-\alpha}$ where $d$ is the distance between $e_{1}$ and $e_{2}$. These higher order terms may also be oscillating. Because $H=0$ the integration contour $C$ in the integral equation fro $\rho(z)$ is a segment of the real line in the additive parameterization. The constant $k_{F}$ is the Fermi-momentum and it is also can be expressed in term of $\rho(z)$. It is also equal to the vertical electrical polarization.

The finite size computations were extended to the case $V \neq 0$ in $[\mathrm{NK}]$. They argued that the complete spectrum of effective $c=1$ conformal field theory is given by

$$
\Delta_{ \pm}=\frac{1}{4}\left(\frac{m^{2}}{g}+n^{2} g \pm 2 n m\right), n, m \in \mathbb{Z}
$$

where $g$ is defined by the Hessian of the free energy and is related to $\alpha$ as $\alpha=\frac{1}{2 g}$.

Combining these results we conjecture that for generic electric fields the asymptotical behavior of correlation functions is still given by formulae (9.1) and (9.2) with $\alpha=\frac{1}{2 g}$

One can show easily that when $\Delta=0$ we have $g=1 / 4$ and the asymptotics (9.1) (9.2) agrees with the results from $[\mathrm{K}]$ on dimer models. 


\subsection{Open problems}

Here we will list some open question about the 6-vertex model and other related models.

- Find the classification of generic singularities of limit shapes. For $\Delta>1$ one should expect the presence of corners as generic singularities of limit shapes.

- Find the scale at which fluctuations near singularities of limits shapes are described by some random process and describe such processes. For example in dimer models such fluctuations near the boundary of the limit shape are described by Airy process, and near a generic cusp are described by the Pearcey process.

One can argue that the same processes should describe correlation functions near similar singularities of the limit shape in the 6-vertex model but this is still a conjecture.

The scaling near the corner singularity seems particularly interesting problem since such singularities do not appear in dimer models.

- Understand the role of integrability of the 6-vertex model in the formation of the limit shape. Here by integrability we mean that the model can be solved by the Bethe ansatz, and that the weights satisfy the Yang-Baxter equation (and therefore transfer-matrices form a commuting family).

- The 6-vertex model is closely related to the representation theory of quantized universal enveloping algebra of $\widehat{s l_{2}}$. It would be extremely interesting to see which aspect of the representation theory of this algebra naturally appear in the limit shape phenomenon and in the scaling of correlation functions near singularities.

- The 6-vertex model has natural generalizations related to other simple Lie algebras. Configurations in these models can be described in terms of $r$ height functions where $r$ is the rank of the Lie algebra. The limit shape in this case is a surface in $\mathbb{R}^{r+2}$. It would be extremely interesting to investigate such systems trying to answer all questions mentioned above.

\section{References}

[AR] D. Allison and N. Reshetikhin, Numerical Study of the 6-Vertex Model with Domain Wall Boundary Conditions, Ann. Inst. Fourier (Grenoble) 55, 1847-1869, 2005.

[AS] M. Abramowitz and I.A. Stegun, Handbook of Mathematical Functions with Formulas, Graphs, and Mathematical Tables, Dover Publications, 1972.

[Bax] R.J. Baxter, Exactly Solved Models in Statistical Mechanics, Academic Press, San Diego, 1982.

[BPZ] N.M. Bogoliubov, A.G. Pronko, and M.B. Zvonarev, Boundary Correlation Functions of the Six-Vertex Model, J. Phys. A 35, 5525-5541, 2002.

[BIR] N.M. Bogoliubov, A.G. Izergin, and N.Y. Reshetikhin. Finite-size effects and infrared asymptotics of the correlation functions in two dimensions. J. Phys. A 20 (1987), no. 15, 5361-5369. 
[Bres] D.M. Bressoud, Proofs and Confirmations: The Story of the Alternating Sign Matrix Conjecture, Cambridge University Press, 1999.

[BS] D.J. Bukman and J.D. Shore, The Conical Point in the Ferroelectric Six-Vertex Model, J. Stat. Phys. 78, 1277-1309, 1995.

[CEP] H. Cohn, N. Elkis, and J. Propp, Local Statistics for Random Domino Tilings of the Aztec Diamond, Duke Math. J. 85, 117-166, 1996.

[CKP] H. Cohn, R. Kenyon, and J. Propp, A variational principle for domino tilings. J. Amer. Math. Soc. 14, 297-346, 2001.

[CP] F. Colomo, and A.G. Pronko,On two-point boundary correlations in the six-vertex model with domain wall boundary conditions. J. Stat. Mech. Theory Exp. 2005, no. 5, 05010, 21 pp.

[DKS] R. Dobrushin, R. Kotecky, and S. Schlosman, Wulff Construction: a Global Shape from Local Interactions, AMS translation series, Providence RI, 104, 1992.

[Elor] K. Eloranta, Diamond Ice, J. Statist. Phys. 96, 1091-1109, 1999.

[FPS] P.L. Ferrari, M. Prähofer, and H. Spohn, Fluctuations of an Atomic Ledge Bordering a Crystalline Facet, cond-mat/0303162.

[HWKK] H.Y. Huang, F.Y. Wu, H. Kunz, D. Kim, Interacting Dimers on the Honeycomb Lattice: an Exact Solution of the Five-Vertex Model, Physica A 228, 1-32, 1996.

[Ize] A.G. Izergin, Partition Function of the 6-Vertex Model in a Finite Volume, (Russian) Dokl. Akad. Nauk USRR, 297, 331-333, 1987.

[JM] M. Jimbo and T. Miwa, Algebraic Analysis of Solvable Lattice Models, CBMS Regional Conference Series in Math. 85, 1993.

[BJMST] H. Boos, M. Jimbo, T. Miwa, F. Smirnov, Y. Takeyama, Hidden Grassmann structure in the XXZ model.hep-th/0606280

[JS] C. Jayaprakash and W.F. Saam, Phys. Rev. B 30, 3916, 1984.

[Ka] P. Kasteleyn, Graph Theory and Crystal Physics, Graph Theory and Theoretical Physics, 43, 1967.

[KPZ] M. Kardar, G. Parisi, and Y.C. Zhang, Phys. Rev. Lett. 56, 889, 1986

[K] R. Kenyon, Height fluctuations in the honeycomb dimer model, math-ph/0405052.

[KO] R. Kenyon, and A. Okounkov, Planar dimers and Harnack curves. Duke Math. J. 131 (2006), no. 3, $499-524$.

[KOS] R. Kenyon, A. Okounkov, and S. Sheffield, Dimers and amoebae. Ann. of Math. (2) 163 (2006), no. 3, math-ph/0311005.

[KW] R. Kenyon, and D. Wilson, Critical resonance in the non-intersecting lattice path model. Probab. Theory Related Fields 130 (2004), no. 3, 289-318.

[KBI] V.E. Korepin, N.M. Bogolyubov, and A.G. Izergin, Quantum Inverse Scattering Method and Correlation Functions, Cambridge University Press, 1993.

[Kor] V.E. Korepin, Calculation of Norms of Bethe Wave Functions, Commun. Math. Phys. 86, 391-418, 1982. 
[KZ] V. Korepin and P. Zinn-Justin, Thermodynamic Limit of the Six-Vertex Model with Domain Wall Boundary Conditions, J. Phys. A, 33, 7053-7066, 2000; Inhomogeneous Six-Vertex Model with Domain Wall Boundary Conditions and Bethe Ansatz, J. Math. Phys. 43, 3261-3267, 2002.

[Kup] G. Kuperberg, Another Proof of the Alternating Sign Matrix Conjecture, Internat. Math. Res. Notices 3, 139-150, 1996.

[Lieb] E.H. Lieb, Phys. Rev. 162, 162, 1967; E.H. Lieb, Phys. Rev. Lett. 18, 1046, 1967; 19, 108, 1967.

[LW] E.H. Lieb and F.Y. Wu, Two Dimensional Ferroelectric Models, in Phase Transitions and Critical Phenomena, Vol. 1, ed. by C.Domb and M.S. Green, 321, Academic Press, London, 1972.

[NK] J.D. Noh and D. Kim, Finite-Size Scaling and the Toroidal Partition Function of the Critical Asymmetric Six-Vertex Model, cond-mat/9511001.

[Nold] I. M. Nolden, The Asymmetric Six-Vertex Model, J. Statist. Phys. 67, 155, 1992; Ph.D. thesis, University of Utrecht, 1990.

[P] K. Palamarchuk, The 6-Vertex Model in Statistical Mechanics, Ph.D. thesis, Univesity of California at Berkeley, 2006.

[PT] V.L. Pokrovsky and A.L. Talapov, Phys. Rev. Lett. 42, 65, 1979.

[Shef] S. Sheffield, PhD Thesis, Stanford Univ. 2003.

[Sm] F. Smirnov, Form factors in completely integrable models of quantum field theory, World Scientific, Singapore 1992.

[SY] B. Sutherland, C.N. Yang, and C.P. Yang, Exact Solution of a Model of Two-Dimensional Ferroelectrics in an Arbitrary External Electric Field, Phys. Rev. Letters 19, 588-591, 1967.

[SY1] B. Sutherland, Phys. Rev. Lett. 19, 103, 1967;

[SZ] O.F. Syljuasen and M.B. Zvonarev, Directed-Loop Monte Carlo Simulations of Vertex Models, cond-mat/0401491.

[Yang] C.P. Yang, Phys. Rev. Lett. 19, 586, 1967

[Yang1] C.N. Yang and C.P. Yang, Phys. Rev. 150, 321, 1966; 150, 327, 1966

[Z] P. Zinn-Justin, Six-Vertex Model with Domain Wall Boundary Conditions and One-Matrix Model, Phys. Rev. E 62, 3411-3418, 2000.

[Z1] P. Zinn-Justin, The Influence of Boundary Conditions in the Six-Vertex Model, cond-mat/0205192.

[Zub] J.-B. Zuber, On the Counting of Fully Packed Loop Configurations. Some New Conjectures, math-ph/0309057. 


\section{A. The Partition Function of the Inhomogeneous 6-Vertex Model}

Here we compute the partition function of the inhomogeneous 6-vertex model with the periodic boundary conditions applying the Bethe Ansatz method. We briefly describe the results and refer the reader to [KBI] for the details about the method.

Bethe Ansatz method works for the inhomogeneous 6-vertex model with the follwing inhomogeneities. One should parameters $\lambda_{1}, \lambda_{2}, . ., \lambda_{M}$ to each row of the grid $L_{N, M}$ and parameters $\mu_{1}, \mu_{2}, . ., \mu_{N}$ to each column of the grid as shown on Fig. 14. Thus, a pair of spectral parameters $\left(\lambda_{i}, \mu_{j}\right)$ is associated to the vertex $(i, j)$ of the grid. Boltzmann weights assigned to this point are:

$$
\begin{gathered}
a_{1}(i, j)=a\left(\lambda_{i}-\mu_{j}\right) e^{H+V}, a_{1}(i, j)=a\left(\lambda_{i}-\mu_{j}\right) e^{H+V} \\
b_{1}(i, j)=b\left(\lambda_{i}-\mu_{j}\right) e^{H-V}, b_{1}(i, j)=b\left(\lambda_{i}-\mu_{j}\right) e^{-H+V} \\
c_{1}(i, j)=c\left(\lambda_{i}-\mu_{j}\right), c_{1}(i, j)=c\left(\lambda_{i}-\mu_{j}\right)
\end{gathered}
$$

where functions $a(\lambda), b(\lambda), c(\lambda)$ describe the parameterization of Boltzmann weights of the homogeneous model.

The partition function of the model with periodical boundary conditions can be written as

$$
Z_{N}=\operatorname{Tr} \prod_{k=1}^{N} T\left(\lambda_{k}\right)
$$

where $T$ is the $2^{N} \times 2^{N}$ row-to-row transfer matrix of the 6-vertex model [Bax]. The raw-to-raw transfermatrix is the trace of the "quantum monodromy matrix":

$$
T\left(\lambda_{k}\right)=\operatorname{tr}_{0} \tau\left(\lambda_{k}\right)
$$

Here $\tau$ is the product

$$
\tau\left(\lambda_{k}\right)=R_{0,1}\left(\lambda_{k}-\mu_{1}\right) \ldots R_{0, N}\left(\lambda_{k}-\mu_{N}\right)
$$

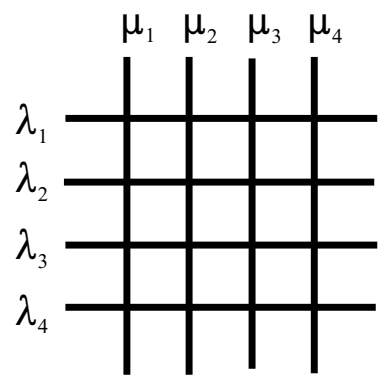

Figure 14: The 4-by-4 square grid with corresponding spectral parameters. 
which acts in $\mathbb{C}^{2} \otimes \mathbb{C}^{2 \otimes N}$. The matrix $R_{0, j}\left(\lambda_{k}-\mu_{j}\right)$ acts as the matrix

$$
\left(\begin{array}{cccc}
a_{1}\left(\lambda_{k}-\mu_{j}\right) & 0 & 0 & 0 \\
0 & b_{2}\left(\lambda_{k}-\mu_{j}\right) & c_{1}\left(\lambda_{k}-\mu_{j}\right) & 0 \\
0 & c_{2}\left(\lambda_{k}-\mu_{j}\right) & b_{1}\left(\lambda_{k}-\mu_{j}\right) & 0 \\
0 & 0 & 0 & a_{2}\left(\lambda_{k}-\mu_{j}\right)
\end{array}\right) .
$$

in the basis $e_{1} \otimes e_{1}, e_{1} \otimes e_{2}, e_{2} \otimes e_{1}, e_{2} \otimes e_{2}$, of the product of the 0 -th and $\mathrm{j}$-th factors in the tensor product. It acts trivially in other factors.

Let us denote the number of arrows pointing down in a row of the grid by $n$. Denote by $\left(\mathbb{C}^{\otimes N}\right)_{n}$ the corresponding subspace in the space of all possible states on the raw of length $N$ of vertical edges. The action of the transfer-matrix preserve these subspaces.

The Bethe Ansatz method gives the following result for the eigenvalues of $T$ :

$$
\Lambda\left(\lambda, u_{1}, . ., u_{n}, H, V\right)=\Lambda_{L}\left(\lambda, u_{1}, . ., u_{n}, H, V\right)+\Lambda_{R}\left(\lambda, u_{1}, . ., u_{n}, H, V\right),
$$

where

$$
\begin{aligned}
& \Lambda_{R}\left(\lambda, u_{1}, . ., u_{n}, H, V\right)=e^{N H+(N-2 n) V} \prod_{l=1}^{n} \frac{a\left(u_{l}-\lambda\right)}{b\left(u_{l}-\lambda\right)} \prod_{j=1}^{N} a\left(\lambda-\mu_{j}\right), \\
& \Lambda_{L}\left(\lambda, u_{1}, . ., u_{n}, H, V\right)=e^{-N H+(N-2 n) V} \prod_{l=1}^{n} \frac{a\left(\lambda-u_{l}\right)}{b\left(\lambda-u_{l}\right)} \prod_{j=1}^{N} b\left(\lambda-\mu_{j}\right),
\end{aligned}
$$

and $u_{i}$ satisfy the Bethe equations

$$
e^{2 N H} \prod_{k=1, k \neq l}^{n} \frac{a\left(u_{k}-u_{l}\right) b\left(u_{l}-u_{k}\right)}{a\left(u_{l}-u_{k}\right) b\left(u_{k}-u_{l}\right)}=\prod_{j=1}^{N} \frac{b\left(u_{l}-\mu_{j}\right)}{a\left(u_{l}-\mu_{j}\right)} .
$$

It follows that the partition function of the model is given by

$$
Z_{N, M}=\sum_{a} \prod_{k=1}^{N} \Lambda_{a}\left(\lambda_{k}, u_{1}, u_{2}, . ., u_{n}, H, V\right) .
$$

where $\Lambda_{a}$ are the eigenvalues of the row-to-row transfer matrix $T$ and the sum is taken over all eigenvalues with their multiplicities.

Thus, in the homogeneous case when all $\lambda_{k}$ are the same the asymptotic of the partition function as $M \rightarrow \infty$ is

$$
Z_{N, M}=d \Lambda_{\max }(N)^{M}(1+o(1))
$$

where $d$ is the multiplicity of the largest eigenvalue $\Lambda_{\max }(N)$ of the row-to-row transfer-matrix on $N$ sites .

Therefore, if we can compute the asymptotic of the largest eigenvalue of the transfer-matrix as $N \rightarrow \infty$ we can find the asymptotic of the partition function in the thermodynamical limit.

Strictly speaking, this logic gives the asymptotic of the partition function in the limit $M \gg N \gg 1$. Under mild assumptions one can argue that the leading term of the asymptotic of free energy is uniform. However, in some cases a resonant phenomenon may occur which will make the leading term of the asymptotic of $Z_{N, M}$ depend on the ratio $N / M[\mathrm{KW}]$. 


\section{B. The Free Energy of the Homogeneous 6-Vertex Model}

In the homogeneous 6-vertex model the Boltzmann weights are the same for all vertices on the grid $L_{N}$. When $\mid \Delta<1$ the functions $a, b, c$ are:

$$
a=\rho \sin (u+\eta), \quad b=\rho \sin (u), \quad c=\rho \sin (\eta) .
$$

Other values of $\Delta$ can b obtained by analytical continuation.

The formula for eigenvalues of the row-to-row transfer matrix given in the previous section becomes

$$
\Lambda^{(n)}(N)=a^{N} e^{N H+(N-2 n) V} \prod_{j=1}^{n} \frac{\sin \left(u_{j}-u+\eta\right)}{\sin \left(u_{j}-u\right)}+b^{N} e^{-N H+(N-2 n) V} \prod_{j=1}^{n} \frac{\sin \left(u_{j}-u-\eta\right)}{\sin \left(u_{j}-u\right)},
$$

where $\left(u_{1}, . ., u_{n}\right)$ are solutions of the Bethe ansatz equations

$$
\left[\frac{\sin \left(u_{j}\right)}{\sin \left(u_{j}+\eta\right)}\right]^{N}=e^{2 N H} \prod_{\substack{k=1 \\ k \neq j}}^{n} \frac{\sin \left(u_{k}-u_{j}+\eta\right)}{\sin \left(u_{k}-u_{j}-\eta\right)}, \quad j=1, . ., n .
$$

Introducing new variables

$$
z_{j}=\frac{\sin \left(u_{j}\right)}{\sin \left(u_{j}+\eta\right)}, \quad j=1, . ., n, \quad w=\frac{\sin (u)}{\sin (u+\eta)}
$$

we get

$$
\Lambda^{(n)}(N)=a^{N} e^{N H+(N-2 n) V} \prod_{j=1}^{n} \frac{1-2 \Delta w+w z_{j}}{z_{j}-w}+b^{N} e^{-N H+(N-2 n) V} \prod_{j=1}^{n} \frac{1-2 \Delta z_{j}+w z_{j}}{w-z_{j}}
$$

where $z_{i}$ satisfy the Bethe equations

$$
z_{j}^{N}=(-1)^{n-1} e^{2 N H} \prod_{k=1}^{n} \frac{1-2 \Delta z_{j}+z_{j} z_{k}}{1-2 \Delta z_{k}+z_{j} z_{k}} .
$$

We want to find the asymptotic of the largest eigenvalue of the transfer-matrix when $N \rightarrow \infty$. We shall do it in two steps. First we will find the asymptotic of the largest eigenvalue among $\Lambda^{(n)}(N)$ in the limit

$$
N \longrightarrow \infty, \quad n \longrightarrow \infty, \quad \frac{n}{N}=\alpha=\text { const }
$$

After this we will find for which $0 \leq \alpha \leq 1$ this eigenvalue is the largest.

\section{The 6-Vertex Model Bethe Equations}

Let us denote

$$
e^{i \theta(z, w)}=\frac{1-2 \Delta z+z w}{1-2 \Delta w+z w}
$$


Then, taking the logarithm of both sides of each Bethe equation (B.1) and dividing by $N$, we get

$$
\ln \left(z_{j}\right)=\frac{n-1}{N} i \pi+2 H+\frac{i}{N} \sum_{k=1}^{n} \theta\left(z_{j}, z_{k}\right)+2 \pi i k_{j} / N, \quad j=1, . ., n,
$$

where $k_{j}$ are integers.

All theoretical and numerical results on the 6-vertex model suggest that the following is true.

Conjecture C.1. In the limit $N \rightarrow \infty$ with $\alpha=\frac{n}{N}$ is fixed the roots of the Bethe equations corresponding to the maximal eigenvalue of the transfer matrix in the subspace $\left(\mathbb{C}^{\otimes N}\right)_{n}$ concentrate on a smooth curve $C$ in the complex $z$-plane. The curve $C$ is symmetric with respect to the complex conjugation $z \rightarrow \bar{z}$.

Conjecture C.2. The maximal eigenvalue of the transfer-matrix in the subspace $\left(\mathbb{C}^{\otimes N}\right)_{n}$ corresponds to $k_{j}=\frac{j}{N}$.

These conjectures can be proven in the free-fermion case $\Delta=0$.

The conjecture (C.1) implies that as $N \rightarrow \infty$ one should expect finite limit density of $z_{j}$ on the contour $C$. If $z_{j} \rightarrow z \in C$ the limit $\rho(z)=\lim _{N \rightarrow \infty} \frac{i}{N\left(z_{j+1}-z_{j}\right)}$ is the value of the limit density function of solutions to the Bethe equations on $C$. Let us denote the endpoints of the contour $C$ by $\xi$ and $\bar{\xi}, \xi=\lim _{N \rightarrow \infty} z_{1}$ and $\bar{\xi}=\lim _{N \rightarrow \infty} z_{n}$.

Taking the limit in the equation (C.2) we obtain the integral equation for $\rho(z)$ :

$$
\ln (z)=2 H-\pi i \alpha+\frac{1}{2 \pi} \int_{C} \theta(z, w) \rho(w) d w+\int_{\xi}^{z} \rho(w) d w .
$$

Here $z \in C$. Differentiating this equation with respect to $z$, we obtain another, more convenient form of this equation:

$$
\rho(z)=\frac{1}{z}+\frac{1}{2 \pi i} \int_{C} K(z, w) \rho(w) d w,
$$

where

$$
K(z, w)=-i \partial_{z} \theta(z, w)=\frac{2 \Delta-w}{1-2 \Delta z+z w}+\frac{w}{1-2 \Delta w+z w} .
$$

The kernel $K$ is singular on two curves

$$
\begin{aligned}
& C_{1}=\left\{z=z_{1}(w)=\frac{1}{2 \Delta-w} \mid \quad w \in C\right\}, \\
& C_{2}=\left\{z=z_{2}(w)=-\frac{1}{w}+2 \Delta \mid \quad w \in C\right\} .
\end{aligned}
$$

and it can be written as

$$
K(z, w)=-\frac{1}{z-z_{1}(w)}+\frac{1}{z-z_{2}(w)} .
$$

Notice that functions $z_{2}(w)=z_{1}^{-1}(w)$, i.e. $z_{1}$ and $z_{2}$ are inverse to each other.

If $\Delta=0$, coutours $C_{1}$ and $C_{2}$ coincide and the kernel is zero. 
Conjecture C.3. If $\Delta \neq 0$, there are two cases:

- Conours $C, C_{1}, C_{2}$ do not intersect each other;

- Contours $C, C_{1}, C_{2}$, all intersect at two points $w_{ \pm}=e^{ \pm i \eta}$ which are the solutions of $w^{2}-2 \Delta w+1=0$ and are conjugate to each other (these two points may coincide in the degenerate case). This is possible only if $\alpha=1$.

The integral equation on $\rho(z)$ can be rewritten as

$$
\rho(z)=\frac{1}{z}+\frac{1}{2 \pi i} \int_{C} \frac{\rho(w)}{z-z_{2}(w)} d w-\frac{1}{2 \pi i} \int_{C} \frac{\rho(w)}{z-z_{1}(w)} d w .
$$

Notice that the contour $C$ can be deformed as long as it does not intersect the curves $C_{1}$ and $C_{2}$.

The contour $C$ is defined by the condition that the form $\rho(z) d z$ has purely imaginary values on the vectors tangent to $C$

$$
\left.\operatorname{Re}(\rho(z) d z)\right|_{z \in C}=0
$$

and by the normalization condition on $\rho(z)$

$$
\frac{1}{2 \pi i} \int_{C} \rho(z) d z=\alpha
$$

The free energy per site is defined as

$$
f(H, V)=-\lim _{M, N \rightarrow \infty} \frac{\log Z_{N, M}}{N M}=-\lim _{N \rightarrow \infty} \max _{1 \leq n \leq N} \frac{\Lambda^{(n)}(N}{N} .
$$

The formula for eigenvaues $\Lambda^{(n)}(N)$ has two terms. Each of them grow exponentially for large $N$. Generically, one of the terms will dominate the other. Taking into account conjectures about roots of Bethe equations in the thermodynamicallimit we obtain the follwoing integral representation for the free energy [LW]:

$$
\begin{aligned}
f= & \min \left\{\min _{\alpha}\left\{E_{1}-H-(1-2 \alpha) V-\frac{1}{2 \pi i} \int_{C} \ln \left(w-\frac{w^{2}-2 \Delta w+1}{w-z}\right) \rho(z) d z\right\},\right. \\
& \left.\min _{\alpha}\left\{E_{2}+H-(1-2 \alpha) V-\frac{1}{2 \pi i} \int_{C} \ln \left(2 \Delta-w+\frac{w^{2}-2 \Delta w+1}{w-z}\right) \rho(z) d z\right\}\right\},
\end{aligned}
$$

or

$$
\begin{aligned}
f(H, V)= & \min \left\{\min _{\alpha}\left\{E_{1}-H-(1-2 \alpha) V-\frac{1}{2 \pi i} \int_{C} \ln \left(\frac{b}{a}-\frac{c^{2}}{a b-a^{2} z}\right) \rho(z) d z\right\},\right. \\
& \left.\min _{\alpha}\left\{E_{2}+H-(1-2 \alpha) V-\frac{1}{2 \pi i} \int_{C} \ln \left(\frac{a^{2}-c^{2}}{a b}+\frac{c^{2}}{a b-a^{2} z}\right) \rho(z) d z\right\}\right\},
\end{aligned}
$$

where $\rho(z)$ is determined bythe integral equation (C.4) with conditions (C.9) and (C.10). 


\section{The Free Energy in the Free-Fermion Case}

The free-fermion curve in the space of parameters of the 6-vertex model is $\Delta=0$. Since in this case $K(z, w)=0$ the integral equation of $\rho$ immediately implies $\rho(z)=1 / z$. The contour $C$ in this case is an arc

$$
z=e^{2 H} e^{i \theta}
$$

where $\theta \in[-\pi \alpha, \pi \alpha]$.

Let us assume that $a>b$. The formula (C.11) for the free energy with fixed polarization $\alpha$ gives

$$
f_{\alpha}=E_{1}-H-(1-2 \alpha) V-\frac{1}{2 \pi} \int_{-\pi \alpha}^{\pi \alpha} \ln \left|\frac{b}{a}-\frac{a^{2}+b^{2}}{a b-a^{2} e^{2 H} e^{i \theta}}\right| d \theta,
$$

Minimizing this expression in $\alpha$ we get the equation

$$
\cos (\pi \alpha)=\frac{a^{2}\left(e^{2 H+2 V}-e^{-2 H-2 V}\right)+b^{2}\left(e^{-2 H+2 V}-e^{2 H-2 V}\right)}{2 a b\left(e^{2 V}+e^{-2 V}\right)}
$$

This defines the critical value $0 \geq \alpha \geq 1$ when the absolute value of the r.h.s is not greater then 1 . Otherwise $\alpha=0$ or $\alpha=1$.

Proposition D.1. The free energy can be written as the following double integral:

$$
f=\min _{0 \leq \alpha \leq 1} f_{\alpha}=-\frac{1}{4 \pi^{2}} \int_{0}^{2 \pi} d \theta \int_{0}^{2 \pi} d \varphi \ln \left|a_{1}-a_{2} e^{i(\theta+\varphi)}+b_{1} e^{i \theta}+b_{2} e^{i \varphi}\right| .
$$

Proof. The proof is computational. For the double integral in question we have:

$$
\begin{aligned}
& \int_{0}^{2 \pi} d \theta \int_{0}^{2 \pi} d \varphi \ln \left(a_{1}+b_{1} e^{i \theta}+\left(b_{2}-a_{2} e^{i \theta}\right) e^{i \varphi}\right)= \\
& 2 \pi \int_{-\theta_{*}}^{\theta_{*}} d \theta \ln \left(a_{1}+b_{1} e^{i \theta}\right)+2 \pi \int_{-\pi+\theta_{*}}^{\pi-\theta_{*}} d \theta \ln \left(b_{2}+a_{2} e^{i \theta}\right),
\end{aligned}
$$

where

$$
\begin{array}{llr}
\theta_{*}=0 & \text { if } \quad & \left|a_{2}-b_{2}\right|>a_{1}+b_{1}, \\
\theta_{*}=\pi \quad \text { if } \quad & \left|a_{1}-b_{1}\right|>a_{2}+b_{2}, \\
\cos \left(\theta_{*}\right)= & \frac{a_{2}^{2}+b_{2}^{2}-a_{1}^{2}-b_{1}^{2}}{2\left(a_{1} b_{1}+a_{2} b_{2}\right)}, \quad \text { otherwise. }
\end{array}
$$

The last equality holds if $\left|a_{2}-b_{2}\right| \leq a_{1}+b_{1}$ and $\left|a_{1}-b_{1}\right| \leq a_{2}+b_{2}$.

We can rewrite $f$ as

$$
-8 \pi^{2} f=8 \pi^{2} \ln \left(\frac{a_{1}+b_{1}+\left|a_{1}-b_{1}\right|}{2}\right)+2 \pi \int_{-\pi+\theta_{*}}^{\pi-\theta_{*}} d \theta \ln \left|\frac{b_{2}+a_{2} e^{i \theta}}{a_{1}-b_{1} e^{i \theta}}\right|^{2} .
$$


We note that the argument of the logarithm under the integral is greater than 1 for any $\theta$ in the domain of integration.

Taking into account the parameterization of Boltzmann weights by electric fields $H$ and $V$ it is easy to see that (D.4) together with the equation for $\theta^{*}$ coincide with the formula for the free energy derived from the Bethe Ansatz.

The double integral formula can be obtained directly from the Pfaffian solution of the dimer model which maps to the 6-vertex model at $\Delta=0$. 